\title{
P53 protein modulates muscle metabolism and generates a non-genetic risk environment that contributes to triggering Amyotrophic Lateral Sclerosis
}

\author{
Águeda Gema Espina-Zambrano ${ }^{*}$ \\ 1 Delegation of Education, Ministry of Education and Sports-Junta de Andalucía. Juan Antonio de Vizarrón, \\ s/n. Isla de la Cartuja 41092 - Seville, Spain; aguedaespina@gmail.com \\ * Correspondence: aguedaespina@gmail.com; Tel.: +34-955-064-000
}

\begin{abstract}
Amyotrophic lateral sclerosis (ALS) is also called "motor neuron disease". In this review, we propose that ALS is not just a neuromotor disorder, but begins as a disorder of P53-modulated skeletal muscle metabolism, leading to failures at the energy state of the cells, incorrect redox states, motor denervation, and a loss of muscle fibers. Motoneurons die as a consequence of the lack of muscular feedback, and the oligomeric TDP43 aggregates progressively and relentlessly lead to mistakes in peripheral immune self-tolerance sustained over time. An effective treatment has not been found for this devastating pathology, as for 152 years the target has not been accurately defined. Scientists and doctors should consider new knowledge regarding ALS and consider immunomodulatory therapies that, based on genetic analysis and symptoms, can be combined with compounds that regulate metabolism and promote the elimination of useless organelles and cells. What if ALS could be cured as a result of seeing motor neuron disease differently? This review aims to develop that goal and change the paradigm of our understanding of ALS.
\end{abstract}

Keywords: ALS; motor neuron disease (MND); metabolism; muscle; TP53; TDP43; immunomodulatory therapies; neuromuscular junction; mitochondria; epigenetic regulation

\section{Introduction}

Amyotrophic lateral sclerosis (ALS), also known as "Lou Gehrig's disease," is the most common neurodegenerative disease of the neuromotor system. It was described for the first time in 1869 by the French neurologist Jean-Martin Charcot, so the history of this devastating and incurable disease goes back more than 150 years. It is characterized by the degeneration of the upper and lower somatic motor neurons that innervate the voluntary muscles, leading to muscle atrophy and weakness of the skeletal muscles. The autonomic nervous system's motor neurons, which are peptide-dependent, are less affected in the gradual degeneration and subsequent death. The outcome is always fatal, with a mean survival of 2-5 years after diagnosis. ALS shows a multifactorial pathogenesis of unknown etiology and currently lacks an effective treatment despite the scientific advances that have occurred in the last decade. Patients diagnosed with possible or probable sporadic ALS do not receive, for the most part, any intervention other than electromyogram tests, blood tests, and magnetic resonance imaging. In these sporadic cases, which are the majority, genetic studies are only carried out in highly specialized ALS units that are not accessible to all patients, and tests are restricted to the study of the most abundant mutations involved in the disease, namely, in genes SOD1, TARDBP, C9orf72, and FUS. Comprehensive genetic studies are never carried out unless requested from private clinics by the patients themselves, even though there are many other genes that are mutated in sporadic ALS. 
Another complication in the diagnosis and treatment of this disease is determining when it detonated in the body of the patient, as according to the latest scientific studies, the observable symptoms, such as stress, inflammation, and muscle fatigue, appear long after the first biochemical and/or physiological imbalances take root. Only by searching for the origin can we approach its multidisciplinary study quickly and efficiently $[1,2,3$, $4]$.

Mitochondria have been shown to be an early target in the pathophysiology of ALS and contribute to the progression of the disease. The interruption of their axonal transport, the excessive generation of reactive oxygen species (ROS), the disorganization of their structure and dynamics, the alterations in mitophagy, the failures in the Krebs cycle and in the oxidative phosphorylation chain, the poor buffering of calcium, and apoptotic activation are directly involved in the pathogenesis of the disease and have been extensively documented in patients with ALS and animal models of the disease. Alterations in energy production are therefore an evident symptom of ALS [5].

This article reviews the literature at the forefront of science worldwide, and we seek to identify an origin of the disease that only a few have suggested for different types of ALS. In addition, we propose new treatments using medicine and physiotherapy for the stabilization and possible reversal of symptoms to mitigate the certainly fatal outcome of the disease. We then detail a novel model of the beginning of ALS that could change the paradigm of this pathology, which has been overlooked due to its low incidence and prevalence $[1,2,3,6,7]$.

\section{ALS is an immunometabolic disease that commences with P53-modulated skeletal muscle energy impairment}

In humans, the muscular system is divided into two distinct categories: smooth and striated muscles. The cellular density, organization, and function of skeletal muscle (SM) require high energy consumption compared to the rest of the body. Striated or skeletal muscles are innervated by the central nervous system (CNS), specifically by $\alpha$-motor neurons, which are cholinergic neurons whose soma are found in the anterior horn of the spinal cord and in the motor nuclei of the cranial nerves. The set formed by an $\alpha$-motor neuron and the skeletal muscle fibers that it innervates constitutes a functional unit called a "motor unit" [8].

In ALS, not all motor neurons (MNs) are vulnerable; slow, fatigue-resistant type I MNs are slow to degenerate, and fast-twitch type II MNs are much more susceptible to apoptosis. Type I MNs are characterized by their aerobic energy production and high mitochondrial density, high activity of oxidative enzymes, high concentration of citochrome c, rich capillary supply, high myoglobin concentration, and low activity of myosin-ATPase. They have a slower conduction speed and lower threshold of excitation to produce an action potential than their counterparts. Conversely, type II MNs are accompanied by anaerobic energy production and are characterized by low mitochondrial density, high glycolytic enzyme activity, poor capillary supply, low myoglobin concentration, and high myosin-ATPase activity. They innervate large muscle fibers that can exert great force in short periods of time by employing anaerobic metabolism. They have high conduction speeds and a high excitation threshold [8].

MN populations that do not have extensive corticospinal entrances, such as MNs within the trochlea and nuclei of Onuf, are resistant to degeneration even when the disease is in its later stages [9]. Nuclei of Onuf MNs innervate striated voluntary pelvic floor muscles, and the neurons themselves are histologically and biochemically comparable to other somatic spinal MNs. However, curiously, these neurons also show some autonomous characteristics since, for example, they receive strong peptidergic innervation. Between the axons of the MNs and the skeletal muscle fibers, a synapse is established 
that has been widely studied as a model of chemical transmission, which is called the neuromuscular junction (NMJ) or motor plate.

Although recent neurophysiological data obtained in ALS patients support early hyperexcitability of cortical MNs, dismantling of the NMJ is one of the earliest anatomical pathogenetic events in ALS. In both ALS patients and murine models of SOD1 disease, dismantling of the NMJ occurred before the degeneration of MNs, when clinical motor signs had not yet been seen $[10,11]$. This indicated that the death of the motor neuron is not, as previously thought, the cause of the dismantling of the NMJ, or at least not the only cause. There seems to exist an inverse process that begins in the skeletal muscle and continues towards synaptic degeneration in the most distal portion of the axon. This novel idea has been supported by research showing that safeguarding the motor neuronal soma did not prevent the loss of the NMJ $[12,13]$.

Although ALS has traditionally been considered an intrinsic disease of somatic MNs, scientific data obtained in recent years have strongly suggested that there are other tissues, such as muscle, vascular, and the organ, involved in the onset and progression of the disease and in energy metabolism; therefore, ALS has a multisystemic pathology. Since glucose is the main circulating energy substrate for muscle and the adult brain, the dysfunction of glucose metabolism inevitably disrupts the normal functioning of the neuromotor system $[1,2,3,4,12,13,14]$.

There are multiple mechanisms that regulate different aspects of skeletal muscle biology, including its development, regeneration, and glucose and oxidative metabolism. Protein arginine methyltransferases (PRMTs) are a family of enzymes that catalyze the methylation of arginine residues in target proteins, and thus mediate a diverse set of intracellular functions that are essential for muscle survival. Indeed, PRMT1 null mice are embryonic lethal. Since their first description in muscle in 2002, studies in a wide variety of experimental models have supported the hypothesis that PRMTs regulate other proteins, such as peroxisome proliferator-activated gamma coactivator (PGC-1 $\alpha$ ), which in turn modulate metabolism and inflammation through the activation of nuclear receptors, transcription factor E2F1, and tumor suppressor protein p53, which ultimately determine the remodeling of the muscle phenotype (i.e., muscle plasticity). Recent studies showing that PRMT function was dysregulated in Duchenne muscular dystrophy, spinal muscular atrophy, and amyotrophic lateral sclerosis have indicated that altering PRMT expression and/or activity could have therapeutic value for neuromuscular disorders (NMD) $[15,16]$.

During environmental changes (strenuous exercise, toxin ingestion, lack of nutrients during long-term forced labor, dehydration, poor sleep, contact with cyanobacteria, latent infections such as Epstein-Barr viral infection or chronic inflammatory demyelinating polyradiculoneuropathy (CIDP), etc.), cells undergo metabolic adaptation through the use of evolutionarily preserved stress response programs. This metabolic homeostasis is exquisitely regulated, and its imbalance could be the basis for many human pathological conditions $[17,18,19]$. The C9orf72 gene, which is related to the most common forms of familial and sporadic ALS, is a key regulator of lipid metabolism under stress. The non-coding repeat expansion leading to loss of function of the protein product C9orf72 leads to an overactivation of starvation-induced lipid metabolism, which is mediated by the deregulated autophagic digestion of lipids and increased de novo fatty-acid synthesis. C9orf72 works by promoting the lysosomal degradation of coactivator-associated arginine methyltransferase 1 (CARM1), also known as PRMT4, which in turn regulates autophagiclysosomal functions and lipid metabolism. In neurons and tissues derived from patients with ALS, a reduction in the function of C9orf72 has been associated with dysregulation in the levels of CARM1, fatty acids, and NADPH oxidase (NOX2). Furthermore, the genetic deletion of Nox2 in the murine SOD1-G93A model of ALS mark- 
edly increased survival. These results revealed a C9orf72-CARM1 axis in the control of stress-induced lipid metabolism and implicated epigenetic dysregulation in devastating human diseases such as ALS [20, 21].

The muscles of whole-body PRMT7 knockout (KO) animals have exhibited decreased ROS and reduced expression of genes such as PGC-1 $\alpha$. In skeletal muscle, PGC- $1 \alpha$ serves as a key regulator of the slow oxidative myogenic program. For example, transgenic overexpression of the coactivator specifically within skeletal muscle results in mitochondrial biogenesis, rapid-to-slow myosin changes, structural and functional alterations in the NMJ, improvements in $\mathrm{VO} 2$ max, and increased endurance capacity [22]. PRMT7 KO mice have shown attenuated endurance exercise capacity, as compared to their wild-type littermates, and decreased energy expenditure. In addition, PRMT7 regulates the slow oxidative phenotype by interacting with the p38/ATF2 pathway and thus improving the expression and activity of PGC-1 $\alpha$ [22].

Many other studies have also shown that PRMT1-mediated arginine methylation regulates nuclear-cytosolic transport of FUS, another ALS-associated protein [23]. While in vivo research clarifying the expression and function of PRMTs in muscle has been limited, recent work clearly demonstrated the emerging importance of this family of enzymes as regulators of skeletal muscle plasticity, and they should be further studied in ALS [24, 25]. In fact, some altered epigenetic profiles of PRMT genes have been detected in the blood and may be useful biomarkers for diagnosis and evaluation of disease or treatment progress [26].

Since 2007, p53 has been known to play a role in cell metabolism, growth, and development, and its involvement in glucose metabolism has recently been demonstrated [27]. Resistance exercise encourages localization of p53 in skeletal-muscle mitochondria, where it stimulates organelle biogenesis [24]. p53 also promotes aerobic metabolism in skeletal muscle, plays a role in muscle differentiation, and may be a therapeutic target for diseases of mitochondrial etiology. Previous studies have revealed the participation of PRMT1 and CARM1 in the activation of p53. More recent research has shown that the PRMT5 protein, as a cofactor of the DNA damage-sensitive coactivator complex that interacts with p53, is responsible for p53 methylation. This methylation affects the specificity of p53 for target genes, and the depletion of PRMT5 triggers apoptosis that is dependent on this tumor suppressor. Therefore, methylation of arginine residues is an underlying mechanism of control during the p53 response [25].

There are close connections between the p53 pathway and pyruvate metabolism. MDM2, an E3 ubiquitin-protein ligase that recognizes the N-terminal transactivation domain (TAD) of the protein, acts as an important negative regulator of p53 both in its degradation and in its transcriptional inactivation. MDM2 and p53 itself are part of a regulatory network in pancreatic beta cells that control pyruvate carboxylase (PC) activity, influencing glucose-stimulated insulin secretion and glucose homeostasis. Important aspects of the metabolic activities of p53 are related to its multiple functions in the transport and storage of lipids; in the biosynthesis of fatty acids and their desaturation; in the metabolism of cholesterol and sphingolipids; and in the oxidation of fatty acids (FAO). Additionally, several studies have documented that p53 interferes with adipocyte differentiation in stressed cells [28, 29, 30, 31, 32, 33].

Previous studies have underscored the importance of p53 in the autophagy network to promoting cell survival. However, p53 can also trigger autophagic cell death under various conditions of severe stress. P53 can regulate mitophagy and macroautophagy, a process that leads to the synthesis of double-membrane vesicles and their fusion to lysosomes to recycle macromolecules and maintain intracellular groups of metabolites. Depending on its subcellular location, its mutational status, and the type of stress, p53 
can inhibit or stimulate autophagy through multiple mechanisms, including the transcriptional control of many genes related to autophagy; the regulation of the kinase mTORC1, which closely controls the autophagic process in accordance with intracellular energy and nutrient levels through regulation of members of the BCL2 family, which also control autophagy; and upon direct interaction with the key autophagic regulator BECLIN $1[34,35]$.

Despite the significant amount of data on this pleiotropic tumor suppressor, there is currently no unifying model that explains how p53 and its multiple regulators coordinate metabolism, but the latest scientific publications have answered questions that are applicable in many physiological and pathological contexts, including metabolic diseases such as diabetes or ALS. P53 is an important effector in many metabolic stress responses that works by controlling the transcription of numerous metabolic genes. Several studies have shown that its DNA-binding properties are directly controlled by intracellular levels of reactive oxygen species (ROS) and by metabolites such as L-serine, all of which would support the theory that p53 is also a metabolic sensor that plays a central role as a mediator in the plastic cell response to redox changes and serine levels. Moreover, its recruitment to chromatin is mediated by the metabolic sensor PKM2, a glycolytic enzyme that has its activity modulated by intracellular levels of serine, and the transcription factor ATF4, which controls the expression of genes involved in the de novo synthesis of serine and serine transport. Whether the activities of other components of the p53 pathway are also directly modulated by metabolites through conformational changes that could affect protein-protein interactions, DNA binding, subcellular localization, and even E3 ligase function, remains to be investigated. It is possible that when cells face a transient decrease in intracellular serine levels, they initially activate de novo serine synthesis by chromatin-bound MDM2 but later induce the p53-mediated repression of PHGDH, an enzyme known as 3-phosphoglycerate dehydrogenase that catalyzes the transition from 3-phosphoglycerate to 3-phosphohydroxypyruvate, which is the concomitant step in the phosphorylated pathway of L-serine biosynthesis. It is also essential in the synthesis of cysteine and glycine, both of which are downstream. This pathway represents the only way to synthesize serine in most organisms, except in plants. Taken together, these convergent lines of evidence indicate that the p53 pathway controls a highly branched metabolic network that is essential for maintaining cellular homeostasis and cannot be ignored in diseases such as ALS [36, 37].

Second, it is interesting to consider these complex metabolic functions from an evolutionary point of view. The observation that p53, and perhaps other key regulators of this pathway, initially favor cell survival under nutrient-deprived conditions has led to the hypothesis that one of its evolutionarily conserved functions was to protect, at both cellular and systemic levels, the body's integrity when access to nutrients was limited. This idea was initially proposed by Murphy et al. in studies aimed at understanding the functional differences of the arginine and proline variants at codon 72 of the TP53 gene. Interestingly, population-based studies and genetically modified murine models have indicated that this single nucleotide polymorphism (SNP) was not associated with a higher incidence of cancer (based on its role as a tumor suppressor par excellence), but rather with a higher body weight and an increased risk of diabetes. As compared to cells expressing the proline variant (P72), those harboring the arginine variant (R72) showed greater cell survival under nutrient-deprived conditions, but not after genotoxic stress. These findings led to the hypothesis that the R72 allele may have been selected in populations in which a better response to nutrient deprivation provided a selective advantage. Notably, some Li-Fraumeni syndrome patients with TP53 germline mutations showed increased mitochondrial oxidative phosphorylation system capacity in their skeletal muscles. These genetic traits confirmed the close links between p53 and metabolism, a role that may have influenced its selection in human populations. The mutation represents only the tip of the iceberg, and future studies may link other components of the p53 
pathway with metabolic disorders with currently unknown etiology and perhaps many types of ALS [38].

Although challenging, systematically characterizing the multiple metabolic defects triggered by the most common genetic alterations of the $\mathrm{p} 53$ pathway should aid the design of streamlined therapies targeting potential metabolic bottlenecks. Furthermore, p53 is abnormally elevated and active in the CNS of patients with ALS, and it is a central regulator that drives neurodegeneration caused by intragenic repeat variants of C9orf72, key in familial and sporadic ALS [30, 31, 32, 33, 34, 39, 40]. Furthermore, Courney et al. demonstrated that neuromuscular junction NMJ loss was a p53-dependent process and this ubiquitous tumor suppressor was a clear effector of axonal and synaptic degeneration in an ALS-like neuropathy [41].

However, it is not clear whether the degeneration of the motor system (MS) in ALS is intrinsically triggered and autonomous or whether the initiating mechanisms of the disease are extrinsic. We hypothesized, in agreement with Martin et al., that skeletal muscle is a primary site of pathogenesis in ALS that triggers degeneration of NMJ by failures in the obtaining of energy by the skeletal muscle, as for instance those that take place in the enzymes of the glycolysis or in the mitochondrial complex I. These energy disruptions generate subproducts (ROS, Lserina) which regulate the action of p53 in the muscle metabolism. If p53 does not correct and adapt the muscle fiber on time, if there is not myophagia or any other process of muscle clearance, then the muscle fiber stops being innervated by its motor neuron. As a consequence, it dies for it misses the feedback of its target (the healthy muscle). This is the way changes would take place given the huge muscle plasticity; thus, there would be loose motor neurons as the neuromuscular junctions would be lost. These synaptopathies would be the beginning of the progressive degeneration of the whole neuromotor system [9].

Some inherited forms of ALS are caused by mutations in the superoxide dismutase-1 (SOD1) gene, which encodes a well-characterized antioxidant protein that is dependent on the copper/zinc balance. In fact, most murine models for ALS were full-length SOD1 mutants. Martin et al. created different types of transgenic mice that expressed the variants of the human SOD1 genes G37R and G93A, the latter of which is the variant most used in ALS murine models, but only in skeletal muscle. The presence of human SOD1 protein (hSOD1) in skeletal muscle was verified by Western blotting, enzymatic activity gels, and immunofluorescence. These mice developed limb weakness and paresis with motor deficits, muscle wasting of the limbs and chest, atrophy of the diaphragm, and life-threatening age-related disease. Murine myofibers developed crystalline inclusions, individualized sarcomere destruction, vesiculating mitochondriopathy, DNA damage, and p53 activation. Satellite muscle cells became apoptotic. The diaphragm developed a severe loss of presynaptic and postsynaptic integrity of the neuromuscular junction, including decreased innervation, loss of synaptophysin, nitration of synaptophysin, and the loss of the nicotinic acetylcholine receptor and scaffold protein rapsyn. Coimmunoprecipitation identified the hSOD1 interaction with rapsyn. The spinal cord of these transgenic mice developed macrosomic atrophy. Spinal MNs formed cytoplasmic and nuclear inclusions; exhibited axonopathy, mitochondriopathy, accumulated DNA damage, activated p53, and cleaved caspase-3; and then died. The mice had a $40 \%-50 \%$ loss of MNs. This work published in Frontiers in Neurology dated December 14, 2020, identified a non-autonomous mechanism for MN degeneration that explained its selective vulnerability as potentially a form of retrograde target-deprived neurodegeneration $[39,42]$.

One of the main events of ALS is weight loss, which has often been studied usingbody mass index (BMI) measurements. As the disease progresses, there is a reduction in the BMI and body fat of the patients. Early insulin resistance and glucose intolerance have also been verified in ALS patients. This insulin resistance, which leads to a decrease 
in the sensitivity of peripheral tissues and limits the entry of nutrients into cells, could participate in the reduction of the BMI observed in patients. Data from the literature have also reported that hypermetabolism affects up to $66 \%$ of patients with ALS and is an early event that persists throughout the course of the disease. Surprisingly, SOD1-G86R and SOD1-G93A mice were hypermetabolic, and this change in their metabolism was already detectable in the clinically asymptomatic stage of the disease, before any motor abnormality. Furthermore, an experimental induction of muscle hypermetabolism was sufficient to cause muscle denervation and the loss of MNs [13]. Therefore, the loss of MNs may be due to the metabolic change that takes place in the innervated muscle.

We propose, therefore, that in the skeletal muscles, ALS alters energy metabolism before any symptoms appear, possibly months or even years prior to diagnosis, and gradually modifies the predominant type of muscle fiber to adapt to the lack of energy in order to obtain it (i.e., muscle starvation); that is, glycolysis decreases while $\beta$-oxidation increases. This likely constitutes an adaptive compensatory mechanism modulated by genes related to p53 to obtain energy when glucose metabolism is cut off and would explain why, in most cases of ALS, patients experience abnormal fatigue and have nocturnal muscle cramps before they feel the need to go to the doctor.

\section{There are many causes of energy failure in ALS}

Inexplicable muscle fatigue, a clear sign [13], may be due to multiple causes, possibly contributing to the different known ALS subtypes in terms of aggressiveness, morphological changes, age of onset, speed of progression, etc. Onset is indisputably influenced by the particular genetic environment of each patient. We list below some of the best described initiators in the literature:

3.1. Mutated genes are involved in the mitochondrial oxidative phosphorylation chain. The main mitochondrial abnormalities found in ALS have to do with the respiratory complexes. Wiedemann et al. reported severe dysregulation of respiratory chain complex I and decreased activity of respiratory complexes I and IV in the muscle of sporadic ALS patients, and in the muscles of murine SOD1-G93A models, presymptomatically $[5,43,44]$.

3.2. Reactive oxygen species (ROS) are very short-lived metabolites produced during oxidative phosphorylation. Under normal physiological conditions, a cell consumes oxygen to produce energy, and at the same time must eliminate the ROS produced through defense mechanisms such as superoxide dismutase (SOD) and antioxidant metalloenzymes. Under conditions of oxidative stress and impaired mitochondrial respiration, large amounts of ROS are produced, leading to cellular damage, such as inflammatory responses, excitotoxicity, protein aggregation, and apoptosis. Furthermore, increased $\beta$-oxidation of fatty acids leads to the generation of lipid byproducts that contribute to lipotoxicity and increased ROS production. Many studies have already shown the implications of oxidative stress for aging and ALS. Abnormally high levels of ROS markers were observed in postmortem fluids and tissues from sporadic ALS patients. In the muscles of SOD1-G86R mice, oxidative stress was observed even before the appearance of motor symptoms and obvious signs of denervation. Likewise, in the muscle of SOD1-G93A mice, SOD1 activity increased throughout the progression of ALS, indicating the presence of oxidative stress in the muscle. Dobrowolnyet al. [45] demonstrated that the muscle expression of the SOD1-G93A mutant was sufficient to induce oxidative damage, muscle atrophy, and the dismantling of the NMJ. Recently, increased ROS production was observed in the muscles of SOD1-G93A mice and in the muscles of wild-type mice with transient overexpression of the SOD1-G93A mutation. The changes in mitochondrial functions depended on the progression of the pathology. The finding 
that the mutation directly contributed to mitochondrial dysfunction long before MN death was surprising [45].

3.3. Mitochondrial functions also progressively deteriorate as the disease progresses, and abnormalities in mitochondrial DNA result in decreased activity of certain enzymes (e.g., NADH and COX). Significant induction of the UCP3 protein has been observed in the muscles of ALS patients and mice. UCP3 is an uncoupling protein expressed mainly in the mitochondria of skeletal muscles, and the overexpression of UCP3 in this tissue would induce increases in lipid oxidation (i.e., $\beta$-oxidation) and energy expenditure. UCP3 overexpression in SOD1-G86R mice has led to the degeneration of MNs, the dismantling of NMJs, and decreased survival [46].

3.4. Environmental toxins such as the non-proteinogenic amino acid $\beta$-N-methylamino-L-alanine (BMAA) from cyanobacteria are capable of forming protein aggregates and the consequent cellular proteotoxic stress. Furthermore, BMAA molecularly competes with the cellular proteinogenic L-serine. Cyanobacteria are abundant in warm, widely fertilized environments such as fields and lawns.

Nitrogen is one of the primary plant nutrients, being the main limiting factor for agricultural productivity. The impacts of excessive nitrogen application are the eutrophication of water, acidification, and toxicity. Cyanobacteria have been studied for years in the literature on motor neuron diseases (MND) due to the enormous increase in the incidence of these pathologies in the places where they appear, starting from those first cases observed in the Chamoru, the indigenous inhabitants of the island of Guam, whose diet was contaminated by cyanobacteria symbionts in the cycads from which they made flour for their sustenance, and by the bats that fed on their seeds [47, 48]. In recent years, conclusive studies have been recorded in European populations exposed to cyanobacteria [49]. Toxins from heavily fertilized lawns where soccer and other sports are played could be one explanation for why there is a significantly higher incidence of ALS among soccer players than among the general population. The age, sex, and race-adjusted incidence and mortality from ALS among all National Football League (NFL) players who debuted between 1960 and 2019 were nearly four times higher than in the general population [50]. Likewise, cases of fulminant deaths have been observed in higher mammals after drinking from warm lakes contaminated with cyanobacteria. These deaths could not be attributed to hepatotoxicity due to the rapidity of their occurrence, and they were most likely due to the many copies of pro-apoptotic genes such as TP53 that they have in their genomes. In addition, such mammals have many skeletal muscle fibers - some of these animals were elephants $[51,52,53,54]$.

3.5. Dysfunction in the pyruvate dehydrogenase enzyme and/or in its metabolic modulators (i.e., kinases and phosphatases) would prevent the passage of glycolysis into the Krebs cycle $[55,56,57]$. A family history of diabetes and/or epilepsy also suggests defects in this enzyme and is correlated with ALS disease (studies of confidential medical reports were obtained from clinical trials and references [58, 59, 60, 61, 62]).

3.6. The inhibition of mitophagy and the accumulation of abnormal mitochondrialbrain-derived neurotrophic factor (BDNF) causes mitochondrial fission and clearance in skeletal muscle through the PRKAA/AMPK-PINK1-PRKN/Parkin and PRKAA-DNM1L/DRP1 MFF pathways [63]. The parkin protein is present in the cytoplasm of most cells, but it is translocated to the mitochondria in those cells that have suffered damage to the organelle. Repositioning of parkin in damaged mitochondria results in the delivery of dead organelles to autophagosomes for degradation (i.e., mitophagy). Depletion of BDNF expression in myotubes reduced fatty-acid-induced mitochondrial fission and mitophagy, which were associated with mitochondrial lengthening and poor lipid management. Muscle-specific BDNF KO mice exhibited defective mitophysis and mitophagy, along with accumulations of dysfunctional mitochondria in 
muscle, when fed a high-fat diet. These animals also exacerbated poor metabolic flexibility and increased insulin resistance. In contrast, consumption of a BDNF mimetic (7,8-dihydroxyflavone) increased mitochondrial content and improved mitochondria and mitophagy in skeletal muscles. Therefore, BDNF is an essential myokine for maintaining mitochondrial quality and function, and its repression could contribute to impaired metabolism in ALS and to the degeneration of MNs that innervate the muscles [64].

3.7. Muscle hypoxia is when mitochondria become sources of intracellular ROS due to alterations in oxidative phosphorylation (OXPHOS). During the adaptive response to hypoxia, mitophagy eliminates mitochondria while stimulating energy production through anaerobic glycolysis, but if this response is insufficient, the mitochondrial network itself can initiate the process of programmed death (e.g., intrinsic apoptosis of p53-dependent mitochondria) [30].

The aforementioned causes would explain the fact that muscle fibers with a low number of mitochondria are the first to degenerate in ALS, as they are energy deficient [9, $65,66]$. Pathological changes in muscle that occur before disease onset and independent of MS degeneration have reinforced interest in studying muscle tissue as a potential target for the administration of ALS therapies. Skeletal muscle has recently been described as a tissue with an important secretory function that is toxic to MNs within the context of ALS. Therefore, a fine balance between the biosynthetic and atrophic pathways is needed to induce myogenesis for the repair of muscle tissue. Compromising these pathways due to primary metabolic abnormalities in the muscle could trigger faulty muscle regeneration and neuromuscular junction restoration, with detrimental consequences for MNs, thereby accelerating the development of ALS. According to the proposal of López-Vales et al., bioactive lipid mediators such as Solutex from Lipinova could be of great utility for promoting muscle regeneration [67]; to such a daily treatment plan, it would be necessary to add a compound such as metformin, an inexpensive drug that has been widely prescribed primarily for type 2 diabetes, has been in clinical use for over 60 years, and has a remarkable safety profile. It directly inhibits mitochondrial complex I, that has been shown to operate incorrectly in ALS, which would make oxidative phosphorylation starts in complex II, and thus it would avoid the bottlenecking of metabolic intermediaries and promote the use of fats as energy substrates. It can also target motoneurons, and stabilizes blood glucose levels [68]. This backward signaling from the muscle sustained over time that causes the death of MNs in ALS in a progressive manner may be delayed or even stopped with the proposed drugs, which have already been deemed to be safe. We may therefore avoid the progression of ALS towards the alteration of peripheral adaptive immunity by TDP43 oligomers. In this direction, the investigations of Pozo et al. [69, 70] continue, whose research also indicates a type of a special autoimmunity that will be discussed later in this paper.

4. The subcellular communication between organelles, especially mitochondrial-associated-membrane (MAMs), modulates ion currents and thresholds of motoneuronal action potentials

For the nerve impulse to be transmitted efficiently at the neuromuscular junction, we know that $\mathrm{Ca}^{2+}$ ions are required. To open a cholinergic synaptic vesicle loaded with the neurotransmitter acetylcholine, it is essential to maintain a minimum concentration of extracellular $\mathrm{Ca}^{2+}$. Only then will the conduction of the nerve impulse be possible, and the acetylcholine released into the synaptic space will be able to bind to the binding sites on postsynaptic receptors (i.e., $\alpha$ subunits). When two acetylcholine molecules simultaneously stimulate the two $\alpha$ subunits, a channel opens in the receptor, allowing the passage of $\mathrm{Na}^{+}$and $\mathrm{Ca}^{2+}$ into the myocyte and the exit of $\mathrm{K}+$. It has been estimated that 400,000 receptors must open to create enough stimulus to generate an action potential that triggers 
muscle contraction. Acetylcholine binds for a very short time to its postsynaptic receptors before being broken down into acetate and choline by acetylcholinesterase.

The denervation of a fast muscle and its reinnervation into slow muscle can change the properties of its motor units. The fast motor units acquire the properties of the slow motor units after denervation/reinnervation and vice versa. This is attributed to the change from phasic activity to the new tonic activity imposed by the MNs that reinforce them. Therefore, the pattern of synaptic activity can vary the types of proteins expressed by muscle fibers. Focusing on ALS, we know that potassium channel expression is markedly reduced in the ventral roots of the spinal cord, which are the efferent motor roots that carry information from the cerebral cortex to the muscle (according to autopsy studies). In line with these findings, elevated potassium channel antibodies were found in a cohort of ALS patients, along with higher mean and abnormal titer levels, as compared to a cohort of patients with peripheral nervous system disorders. These results suggest the possibility of a subset of ALS patients where autoimmunity could play a role in the development and progression of the disease [71]. The increased sodium and decreased potassium currents, pathological findings, decreased expression of the potassium channel in the motor axons of ALS, and serological study, together with the presence of antibodies against the potassium channel in patients with ALS, support our interpretation that the pattern of synaptic activity in ALS can change the types of proteins expressed in muscle fibers; that is, there would be an adaptive mechanism of muscle plasticity at the beginning and during the progression of the disease [72].

The main adaptation responsible for the increase in muscular endurance is an increase in the number of mitochondria. However, this adaptation occurs too slowly to provide a survival advantage when there is a sudden change in the environment that requires prolonged exercise. Therefore, there is another more rapid adaptation, down-regulation of the expression of glycogenolytic and glycolytic enzymes in the muscle, which mediates a deceleration in both the depletion of muscle glycogen and the accumulation of lactic acid. This adaptation is mediated by PGC-1 $\alpha$ [73].

In asymptomatic mice with ALS, the muscle cell mitochondria were no longer able to regulate calcium signaling around the NMJ, and an excessively high concentration of calcium in the cytosol may have contributed to the progression of muscle atrophy. These data confirmed a close link between mitochondrial dysfunction and calcium dysregulation. The latter would consequently cause a defect in the mitochondrial respiratory chain, triggering a vicious cycle. This is where ER and Golgi membranes, specifically MAMs, come into play. The ER comprises more than half of the total amount of cell membrane and occupies approximately $35 \%$ of the cytoplasmic volume. However, the intracellular membranous space is more extensive in secretory cells and cells with extended neural processes such as axonal-type terminals, which have developed ER even in distant neurites. This is due to the fact that increased synthesis of peptides and other secretory molecules, their accumulation in the ER and vesicles, and $\mathrm{Ca}^{2+}$-dependent polarized vesicular release require tightly coupled interactions with mitochondria, the Golgi apparatus, and other organelles. For this reason, the ER has been called a "neuron within a neuron" [23].

The mitochondria play a fundamental role in calcium homeostasis. The absorption of this ion has physiological consequences of great importance for muscle cells, in addition to preventing signaling cascades from firing at certain times by reducing the concentration of calcium in the cytoplasm. Free calcium in the mitochondrial matrix is known to increase the ATP synthesis rate by modulating the activity of various enzymes that have products of the Krebs cycle as substrates. Therefore, the flow of electrons is controlled by creating a very large transmembrane potential. This represents a strong net force that draws the cation into the mitochondrial matrix. The process of calcium uptake by the mitochondria must be finely regulated; if calcium enters the matrix continuously, 
there is an overload that causes energy losses, and morphological alterations that promote the release of apoptotic factors or produce necrosis.

Rizzuto and Mootha laboratory team discovered the system that allows the divalent cation to enter the mitochondrial matrix: the mitochondrial calcium uniporter (MCU) [74]. This system exhibits low activity when calcium concentrations are at rest ( 100 nM). The calcium-concentration-dependent MCU activity is regulated by the elements associated with the transporter and that form a multiprotein complex consisting of MICU1 and MICU2. These elements form heterodimers that do not allow the MCU to open when the calcium concentration is basal. However, when the calcium concentration increases, the dimer cooperates and allows the passage of said ion towards the matrix of the mitochondria.

Patron et al. [74] demonstrated that MICU1 and MICU2 have antagonistic regulatory activities. MICU2 is the element that regulates the opening function of the complex, and MICU1 is necessary for MICU2 to perform its function, since when MICU1 is not present, the dimer cannot be formed. In the opposite case, when MICU2 is not expressed, MICU1 homodimers are formed, which allow greater flow of $\mathrm{Ca}^{2+}$ to the interior because there are then two activating elements. Indicating that MICU2 is the element responsible for the regulation of calcium entry into the mitochondria conflicted with the previous concept that MICU1 was responsible for both the positive and negative regulation of the MCU. Therefore, MICU2 was suggested to be an important factor for optimal cellular performance that prevents the continuous accumulation of calcium in the mitochondrial matrix [75]. Dafinca et al. demonstrated in iPSC-MN by RNA sequencing that both C9ORF72 and TARDBP have upregulated Ca2+-permeable AMPA and NMDA subunits and impaired mitochondrial $\mathrm{Ca}^{2+}$ buffering due to an excess of MICU1, indicating that impaired absorption of mitochondrial $\mathrm{Ca}^{2+}$ contributes to glutamate excitotoxicity and is a shared feature of NMs with C9ORF72 or TARDBP mutations [76]. Matteucci et al. supported a model in which the E3 ubiquitin ligase parkin participated directly in the selective regulation of MICU1, and asa gatekeeper of MICU2 MICU2 stability was affected by parkin overexpression, probably as a consequence of increased MICU1 degradation, indicating that the loss of parkin's function could contribute to the deterioration of the ability of the mitochondria to handle $\mathrm{Ca}^{2+}$ [77]. We proposed that the overexpression of the parkin protein, especially through its ubiquitin ligase activity, favors neuroprotection after uncontrolled dysregulation of mitochondrial $\mathrm{Ca}^{2+}$ levels.

MAM collapse is a common pathogenesis mechanism in ALS linked to SIGMAR1, SOD1, VAPB, TARDBP, and FUS [78, 79]. When analyzing ER mitochondria calcium cycle dynamics (ERMCC) in murine model swith hSOD1-G93A (overexpressing mutant) $\mathrm{MNs}$ - as a model for ALS - with sub-sector resolution, using fluorescent calcium imaging, and comparing vulnerable MNs and non-neurons from hSOD1-G93A mice with their non-transgenic littermates, decelerated clearance of cytosolic calcium was associated with hSOD1-G93A. While both the non-transgenic MNs and the G93A mutants showed high absorption of mitochondrial calcium by the mUP uniporter, the mitochondrial calcium extrusion system was disrupted in the presence of hSOD1-G93A. Furthermore, ER calcium uptake by the sarcoplasmic reticulum ATPase (SERCA) was increased in mutant MNs compared to wild type MNs. In survival tests using SERCA inhibitors in mutant mice, it was shown that the endoplasmic reticulum proteins associated with ALS in denervated skeletal muscle explained the course of the disease from the contracted muscle to the MN.

Levels of VAPB, a type IV membrane protein found in plasma and intracellular vesicle membranes that is involved in vesicle trafficking, were decreased in cells from patients with sporadic ALS, and in a murine model of familial ALS caused by a gene unrelated to VAPB. Considering the recent findings on ER-mitochondrial contacts me- 
diated by VAPB-PTPIP51 and on the role of $\mathrm{Ca}^{2+}$ in mitochondrial energy metabolism, it has been investigated whether chronic depletion of VAPB in MN-like NSC34 cells affects mitochondrial function. Borgese et al. in July 2021 [80] found that this cell line has been widely characterized, and alterations have been seen in the homeostasis of phosphoinositides, which act as second messengers in the signal transduction of the cells and the elongation of neurites. Furthermore, a decrease in the uptake of the mitochondrial membrane potential sensor tetramethylrhodamine methyl ester perchlorate (TMRM) has been observed in the mitochondria of VAPB-depleted cells, suggesting reduced oxidative phosphorylation. This observation agrees with the observations of reduced ATP production via oxidative phosphorylation in neuronal cell lines and primary cortical neurons under conditions in which the ER-mitochondria contacts were diluted.

The relevance of these findings to ALS has been reinforced by computational models that predict that even small decreases in ATP availability can disrupt neuronal ion homeostasis and functionality. Importantly, VAPB-PTPIP51-mediated contacts were present in nerve endings, and the silencing of VAPB or PTPIP51 in primary cultures of hippocampal neurons reduced ER-mitochondrial contacts concomitantly with synaptic activity [80]. Using MAMtrackers with a library of expression plasmids of genes causing ALS, it was observed that $76 \%(16 / 21)$ of the genes altered the integrity of MAM. These results suggest that MAM disruption is a common pathological feature in ALS and that VAPB could transiently interact with hyperpolarization-activated cyclic nucleotide-driven $(\mathrm{HCN})$ channels 1 and 2 in the ER, facilitating the assembly of subunits into a competent tetramer for export to the cell surface.

We know that these HCN channels play a crucial role in the spontaneous firing of the action potentials of neurons. Therefore, VAPB would act as an intramembrane chaperone necessary for synaptic transmission [80]. We proposed that the action potential threshold to trigger depolarizing discharges in MNs falls as a compensatory process as ALS (i.e., hyperexcitability) progresses; hence, the hands, feet, extremities, and in general, the skeletal muscles of patients, remain rigid and seized, as if they have received large, continuous electric shocks that have been maintained over time. As they a suitable for high-throughput assays, MAMtrackers may be valuable tools to better understand MAM dynamics in ALS and other neurodegenerative diseases. Therefore, elucidating the structure and function of MAMs may be a novel strategy for the treatment of ALS [81, 82, 83].

\section{The chaperone SIGMA1R is a key regulator in TDP43 proteinopathy}

Sigma-1 receptor (S1R) is an ER transmembrane molecular chaperone that modulates the activity of multiple effector proteins. S1R associates with cholesterol through an intracellular domain. Vladimir Zhemkov et al. found recently that the association of S1R with cholesterol induces the formation of cholesterol-enriched microdomains in the ER membrane that could retain secreted and signaling proteins [84]. Zhemkov et al. proposed that S1R agonists allow the disassembly of cholesterol-enriched microdomains and the release of accumulated proteins such as ion channels, signaling receptors, and ER-trophic factors [85, 86].

Spinal cord MN activation occurs, in part, through large ventral horn cholinergic synapses called C-boutons/C-terminals. In ALS, the chronic excitation of the MNs and alterations in the C-terminals have been observed. S1R plays an important role in the regulation of $\mathrm{MN}$ function in that high levels of S1R are localized in subsurface cisterns of the postsynaptic endoplasmic reticulum (ER) that contain type 2 muscarinic acetylcholine receptors (M2AChR), potassium channels that are activated by calcium (Kv2.1), and slow potassium channels (SK). An increase in action potential frequency in murine S1R-KO $\mathrm{MNs}$ indicated a critical role for S1R as a "brake" on motor neuron function, possibly 
through calcium-dependent hyperpolarization mechanisms involving calcium channels and potassium, as mentioned above. The longevity of SOD1-S1R-KO mice was significantly reduced, as compared to SOD1-WT controls [86].

In this sense, the role of the $\mathrm{Ca}^{2+}$-dependent autophagic pathway in ALS has been demonstrated through the use of the $\mathrm{Ca}^{2+}$ blocker, L-type channel called verapamil. Verapamil has been found to significantly delay disease onset, prolong lifespan, and extend disease duration in SOD1-G93A mice. Furthermore, verapamil administration improved motoneuronal survival and improved skeletal muscle denervation in SOD1-G93A mice. Furthermore, verapamil significantly reduced SOD1 aggregation and improved autophagic flow, which could be mediated by the inhibition of calpain-1 (i.e., a stomach and muscle-specific intracellular cysteine protease) in the spinal cord marrow of these transgenic mice. Furthermore, verapamil reduced ER stress and suppressed glial activation in SOD1-G93A mice. Collectively, these data indicated that verapamil was neuroprotective in the ALS murine model and that the $\mathrm{Ca}^{2+}$-dependent autophagic pathway is a possible therapeutic target for the treatment of ALS [87].

Overexpression of the S1R-E102Q mutant promoted dissociation of the membrane protein from the ER and cytoplasmic aggregation, which in turn impaired mitochondrial ATP production and proteasomal activity. Under ER stress conditions, wild-type S1R overexpression suppressed stress-induced mitochondrial injury, whereas S1R-E102Q overexpression aggravated mitochondrial damage and induced autophagic cell death. Furthermore, cells that overexpress S1R-E102Q showed aberrant foreign-nuclear localization of the DNA-binding protein TDP-43, a condition exacerbated by ER stress. Treatment of cells with a mitochondrial $\mathrm{Ca}^{2+}$ transporter inhibitor $(\mathrm{Ru} 360)$ mimicked the effects of S1R-E102Q overexpression, indicating that aberrant S1R-mediated mitochondrial $\mathrm{Ca}^{2+}$ transport likely underlies the foreign localization of TDP-43, segregation in inclusion bodies, and ubiquitination. Interestingly, the enhancement of ATP production promoted by methyl pyruvate (MP) treatment relieved the deterioration of the proteasome and the foreign localization of TDP-43 caused by overexpression of S1R-E102Q [88].

Finally, in relation to S1R, we must emphasize that a new line of zebrafish deficient in said transmembrane protein has been characterized, presenting visual and locomotor deficiencies in comparison with the wild line. A critical role for S1R in ER stress pathways and mitochondrial activity has been demonstrated. Using qPCR to analyze the gene response to unfolded proteins, it has been observed that loss of S1R leads to alterations in the bioenergetics of mitochondria-decreased basal, ATP-linked, and non-mitochondrial respiration, and the same after tunicamycin challenge (an antibiotic mixture that causes cell cycle arrest in the G1 phase that has been used as an experimental tool to induce the unfolded-protein response). In conclusion, this new zebrafish model confirmed the importance of S1R activity in ER-mitochondria communication and in stopping proteotoxic stress [89].

\section{The immune activation spreads like prions throughout all the neuromotor system}

Activation of the microglial inflammasome NLRP3 has emerged as a key contributor to neuroinflammation during neurodegeneration. Aggregates of pathogenic proteins such as $\beta$-amyloid and $\alpha$-synuclein trigger microglial activation of NLRP3, leading to caspase- 1 activation and IL- $1 \beta$ secretion. Both caspase- 1 and IL- $1 \beta$ contribute to disease progression in the murine ALS SOD1-G93A model, suggesting a role for microglial NLRP3. In knock-in SOD1-G93A mice with the Nlrp3-GFP gene that expresses the microglial NLRP3, it was shown that both aggregated and soluble SOD1-G93A activated the inflammasome in the primary microglia, leading to caspase- 1 cleavage and IL-1 $\beta$ and IL-1 $\beta$ secretion in a dose- and time-dependent manner. Importantly, SOD1-G93A was 
unable to induce IL-1 $\beta$ secretion from microglia deficient in Nlrp3 or pretreated with the specific NLRP3-inhibitor MCC950, confirming NLRP3 as the key inflammatory complex that mediated microglial IL-1 $\beta$ secretion induced by SOD1. Microglial upregulation of NLRP3 was also observed in the ALS murine model TDP-43-Q331K, and wild-type and mutant proteins TDP-43 were also capable of activating microglial inflammasomes in an NLRP3-dependent manner. The generation of ROS and ATP were mechanically identified as key events required for SOD1-G93A-mediated activation of NLRP3. Taken together, these data demonstrate that ALS microglia expressed NLRP3, and that ALS pathological proteins activated the NLRP3 microglial inflammasome. Therefore, inhibition of NLRP3 may be a potential therapeutic approach to arrest microglial neuroinflammation and ALS disease progression [70, 90, 91, 92].

It is striking that the proteinopathy that characterizes ALS is due to the accumulation of TDP-43 aggregates. This protein is the product of another mutated gene in ALS, TARDBP. Under normal conditions, its location is nuclear, but in ALS and other neurodegenerative diseases (albeit to a lesser extent in the latter), the protein passes into the cytosol and self-aggregates into oligomers that can be soluble and affect synaptic function. Maintaining a balance between TDP43 production and clearance is essential to preserving brain health, as accumulations of TDP43 aggregates will lead to increased oligomer production and neurotoxicity. In recent years, the role of inflammation in ALS has gained relevance. Mitophagy is a vital form of autophagy for the selective removal of dysfunctional or redundant mitochondria. Much evidence shows the elimination of dysfunctional mitochondria to be a powerful means employed by autophagy to keep the immune system in check. The mitophagy process can restrict inflammatory cytokine secretion and directly regulate mitochondrial antigen presentation and immune-cell homeostasis. There has been direct and indirect evidence linking mitophagy to inflammation and autoimmunity, which underly the pathogenesis of autoimmune diseases. MTAP appears to be inhibited by mitophagy, directly linking mitophagy with adaptive immunity $[30,31,32,33,34]$.

TDP43 oligomers are excreted and spread to neighboring cells through a mechanism termed "prionic". They interact with the microglia as part of their regular clearance process, and this triggers the activation of immunity and the secretion of pro-inflammatory cytokines and chemokines that recruit more microglia and astrocytes to the site of inflammation [92]. This disproportionate release of pro-inflammatory mediators has been proposed as a contributor to neurodegeneration [94, 95]. TDP43 oligomers were found in the plasma of ALS patients and activated not only innate but also peripheral immunity $[69,94]$. Not surprisingly, TDP43 increased mitochondrial fusion, inhibited mitophagy, regulated fat deposition and glucose homeostasis [97], and induced the p53-mediated cell death of cortical progenitors and immature neurons [98]. Once again, the discovery of the new role of the tumor suppressor p53 in the regulation of glucose biosynthesis through the direct inactivation of glucose-6-phosphate dehydrogenase has proven vital [98, 99]. Taken together, these data have indicated synergy between TDP43, p53, and inflammatory cytokines that may start early in the disease and would allow treatment, which has already been proposed for another proteinopathies, such as Alzheimer's disease [100], in the first stages of ALS with the therapeutic plasma exchange (TPE) technique. TPE has been used in neurology for many years, and not all the indications for which it is performed are mediated by autoantibodies, which are not negligible in ALS either [71, 72]. Some of the diseases for which it has been administered, such as multiple sclerosis, are mediated by neuroinflammation. Therefore, the goal of TPE is to remove harmful cytokines, chemokines, and other substances from the blood that can degenerate MNs after crossing the blood-brain barrier (BBB). Peripheral immune cells directly cross or indirectly transmit signals through the $\mathrm{BBB}$ to modulate or interfere with brain function. The pleiotropic properties of albumin, the main extracellular antioxidant with immunomodulatory properties, could play a key role if used as a replacement fluid in the PE-based 
therapeutic strategy for the treatment of ALS [101]. It seems paradoxical that technology developed for other neurodegenerative diseases has not yet been applied to a fatal disease without treatment, ALS, when in the literature there has only been one study, from 1980, suggesting that the technique would be ineffective. Only four patients were involved, and their genetic backgrounds and medical histories were not noted; however, according to the limited-sample study, they experienced some improvement after the first plasma exchange [102].

However, given the significant inflammatory load that occurs after the onset of ALS, especially in those with a rapid progression and those with the bulbar type, it will be necessary, in addition to all the above, to consider other therapies, such as tocilizumab, since it is safe and well-tolerated in patients with ALS and high levels of mRNA of inflammatory markers [103]. Inflammation plays a crucial role in the pathogenesis of cerebrovascular accidents, and there have been many cases of stroke associated with ALS. Until very recently, strokes were thought to be a risk factor for the disease and not a consequence. Studies such as those by Anna Manberget al. [104] found that the variability of the sporadic progression of ALS may depend on less defined contributions from the glia and blood vessels. Patients with sporadic ALS have presented patterns of cellular activity, consistent with two murine models, in which vascular cell gene enrichments preceded the microglial response. In particular, during the presymptomatic stage, perivascular fibroblast cells showed the strongest genetic enrichments, and their marker proteins osteopontin (OPN) or SPP1 and COL6A1 accumulated in enlarged perivascular spaces in patients with sporadic ALS [104, 105].

New evidence has also indicated the role of OPN in skeletal muscle diseases. Osteopontin has been described as a component of the inflammatory environment in dystrophic and injured muscles and has also been shown to increase diaphragm-muscle healing in aged dystrophic mice. In a recent study, OPN has been identified as a determinant of the severity of disease in patients with Duchenne muscular dystrophy. In addition, a mutation in the promoter of the OPN gene, which causes low levels of expression of the protein, has been associated with a decrease in age until loss of ambulation and muscle strength in patients with other skeletal muscle diseases [104, 106, 107].

The etiology and pathophysiological mechanisms of inflammatory neuropathies are only partially understood, but it is likely that humoral and cellular immunity have roles in their pathogenesis. Autoantibodies against peripheral nerve molecules, such as gangliosides or proteins of the ganglion of Ranvier, have been described, allowing the identification of subgroups of patients with specific clinical phenotypes. Although the exact mechanisms underlying the development of immunopathology remain unknown, immune-mediated neuropathies are considered treatable. The main challenge in successfully selecting immunotherapy has been the great variability in underlying pathobiology, leading to variable clinical presentation and disease course. As in classic CIDP pathology, ALS is associated with macrophage and T-cell infiltration of peripheral nerves. It is not clear how the breakdown of the blood-brain barrier BBB occurs in CIDP, but in animal models, it is one of the first events in nerve inflammation and may be mediated by inflammatory molecules released by inflammatory cells in the peripheral immune compartment. This phenomenon can be triggered by an autoimmune attack against the putative antigen, but allows access of autoantibodies, macrophages, and other immune mediators to the endoneurial space to cause nerve damage.

Recent findings by Pozo et al. point to Th17 cells (i.e., T-helper 17 cells) among peripheral blood mononuclear cells (PBMC) as those most correlated with ALS. Th17 cells are potent inducers of autoimmune diseases through the activation of inflammatory mediators, angiogenesis, and the induction of immune-cell activation. In fact, elevated levels of Th17 cells have been associated with other inflammatory autoimmune diseases, 
such as multiple sclerosis and systemic lupus erythematosus, and could also be important in the neurological progression and rapid spread of ALS pathogenesis [69].

\section{Proposed therapies for the treatment of ALS}

In addition to those already cited in this review of the literature, including such novel proposals as plasma replacement therapy with albumin, administration of intravenous immunoglobulins (IVIg), and the daily administration of metformin with Lipinova's Solutex, additional therapeutic approaches warrant consideration, especially given the paucity of choice in ALS treatments. ALS has various types with particular presentations and complications, though they all result in the same progression: muscle energy failure and sustained motor neuronal apoptosis until the death of the patient. Therefore, we propose an individualized therapeutic approach. The preliminary data of each patient will determine the most appropriate combination of compounds, and a complete genetic analysis clarifying which genes may be failing will be required. To date, the generic treatment of ALS with riluzole has never been sufficiently studied in humans with ALS, since negative controls were impossible and the populations of patients under study have had ALS of different types, progression levels, times of diagnoses, etiology, genetics, etc. Riluzole can cause liver damage, the extent of which can vary, and it does not make the disease chronic; it only lengthens it for a few months. We suggest eliminating it as a first-line treatment both due to its unsatisfactory outcomes and due to it being counterproductive when administered together with those treatments proposed in this paper.

\subsection{Withaferin $A$}

The highly innocuous withaferin A reduces SOD1 aggregates in murine ALS models, extends SOD1's useful life, and selectively inhibits the activation of NF-Kb, which induces p53-dependent apoptosis, thereby avoiding apoptosis in the event of failure in organelle autophagy [108].

\subsection{Carbamazepine vs. verapamil}

Carbamazepine (CBZ) is a well-known antiepileptic drug that has been used in clinical practice for more than four decades. CBZ has been reported to stimulate autophagy by decreasing the intracellular level of inositol, and long-term treatment with CBZ exhibited a protective effect in a murine model of Alzheimer's disease, possibly through improved autophagic flux [109]. Treatment with CBZ reduced MN loss by approximately $46.6 \%$ and improved altered muscle morphology and NMJs. Furthermore, a study of the mechanism revealed that CBZ treatment activated autophagy through the AMPK-ULK1 pathway and promoted the removal of mutant SOD1 aggregation [109, 110]. Verapamil blocks calcium channels, improves skeletal muscle innervation and motor neuronal regeneration, reduces SOD1 aggregation, and suppresses glial activation. Verapamil has been used with success in heart disease and hypertension and would be a fantastic clinical trial candidate for treating ALS, all the more so given its success in trials for diabetes [38]. However, the use of CBZ together with verapamil is not recommended.

\subsection{Edaravone}

Widely used for ALS, edaravone has not provided noticeable results when combined with riluzole, the only drug previously approved by the U.S. Food and Drug Administration (FDA) for ALS. The deterioration of mitochondrial oxidative phosphorylation in skin fibroblasts from patients with SALS and FALS was improved by in vitro treatment with ROS scavengers; therefore, we suggest oral edaravone and an antioxidant diet [111].

\subsection{Trehalose}


Autophagic markers have been observed as upregulated in both MNs from ALS patients and in animal models. In addition, several ALS-causing genes have been identified - such as optineurin (OPTN) [112, 113, 114]; sequestration-1, also known as ubiquitin-binding protein p62 (SQSTM1); valosin-associated protein (VCP), containing a DNA-PK, and it regulates its degradation based on ubiquitin-proteasome; and the subunit 1 of dynactin, which collaborates in bidirectional intracellular transport by binding to dynein (DCTN1) - which play important roles in the autophagy system. Growing evidence has indicated that autophagy activation may play a protective role in animal models. OPTN suppression caused neuronal cell death through the nuclear factor kappa-light-chain-enhancer of activated B cells (NF-Kb) pathway [115]. Mutant SOD1 aggregates related to ALS interfered with mitophagy by sequestering OPTN from the autophagy receptor [116, 117]. Trehalose has already been widely studied by Hetz et al., and it may have potential when administered together with other compounds for the activation of autophagy in those patients who have favorable genetics. Similar to metformin, it may be effective for fatty liver disease, which occurs in a large number of ALS patients [118].

\subsection{L-serine amino acid}

The global development of cyanobacterial blooms has increased significantly in marine and inland waters over the last century due to the eutrophication of water. This phenomenon is characterized by the ability of planktonic cyanobacteria to synthesize gas vesicles that allow them to float in water columns. In addition, the benthic cyanobacteria that thrive at the bottoms of lakes, rivers, and coastal waters form dense mats near the shore. The massive proliferation of cyanobacteria is harmful to animal and human health, particularly due to the ability of certain strains of cyanobacteria to produce hepatotoxic and neurotoxic compounds. Cholinergic synapses and voltage-gated sodium channels are the targets of choice for cyanobacterial neurotoxins. Anatoxin-a and homoanatoxin-a are agonists of nicotinic acetylcholine receptors. Anatoxin-a is an irreversible acetylcholinesterase inhibitor. Saxitoxin, kalkitoxin, and jamaicamide are voltage-gated sodium channel blockers. Antillatoxin is an activator of these channels. Furthermore, the neurotoxic amino acid BMAA has been shown to be produced by various taxa of cyanobacteria. In vivo and in vitro evidence has suggested a link between the ingestion of BMAA and the development of the amyotrophic lateral sclerosis-parkinsonism-dementia complex, a neurodegenerative disease that was described for the first time in the indigenous people of Guam. Supplementation with L-serine would be of interest in those cases of ALS where there is suspicion of environmental contamination by cyanobacteria $[49,51,52$, 53]. In addition, as we said above, the amount of L-serine and ROS regulates the plastic metabolic response by p53 in muscle $[36,37]$.

\subsection{Thiamine or vitamin B1}

Pyruvate dehydrogenase deficiency presenting as isolated dystonia induced by paroxysmal exercise was successfully reversed with thiamine/vitamin B1 supplementation [119]. By coupling glycolysis and the mitochondrial tricarboxylic acid cycle, the pyruvate dehydrogenase (PDH) complex (PDHC) is highly sensitive to cellular demands through multiple mechanisms, including the phosphorylation of $\mathrm{PDH}[57,58]$. PDHC also produces acetyl-CoA for the acetylation of proteins involved in the circadian regulation of metabolism. Thiamine diphosphate (vitamin B1) (ThDP) is known to activate $\mathrm{PDH}$ as a coenzyme and inhibitor of PDH inactivating kinases. The molecular mechanisms that integrate the role of thiamine-dependent PDHC in general redox metabolism underlie the physiological fitness of a cell or organism. There are diurnal and thiamine-dependent changes in the function, expression, and phosphorylation of PDHC in the rat brain, and its impacts on protein acetylation and metabolic regulation have been evaluated. The morning administration of thiamine significantly regulated the phosphorylation of PDH at both the Ser293 and SIRT3 protein levels [56], but these effects were not observed with evening administration. This action of thiamine abrogates the 
diurnal dependent changes in brain PDHC activity and mitochondrial acetylation, inducing the diurnal differences in cytosolic acetylation and total brain protein acetylation. Screening for diurnal dependence among central metabolic enzymes and proteins of thiol/disulfide metabolism revealed that thiamine also eliminates daily changes in malate dehydrogenase activity, as opposed to PDHC activity. A correlation analysis indicated that thiamine abrogated the strong positive correlation between total acetylation of brain proteins and PDHC function. Simultaneously, thiamine increased the relation between the expression of PDHC components and total acetylation of the SIRT2 protein level. These effects of thiamine on the brain acetylation system changed the metabolic impact of acetylation. Therefore, the diurnal action of thiamine on PDHC and SIRT3 may be of therapeutic importance for correcting disturbed diurnal metabolic regulation and defects after exercise. Furthermore, the interaction of ThDP with p53 and PDH kinases indicates that this vitamin may be a suitable and inexpensive candidate for daily supplementation, even more so if it is combined with melatonin for regulation of the circadian rhythm in night workers and other patients unable to regulate their wake/sleep cycles. Furthermore, melatonin has positive effects on the regulation of autophagy [120].

\subsection{Vitamin B12/vitamin D/folic acid}

Some types of ALS commence with a diet low in animal protein for extreme weight-loss therapies (according to confidential patient data). The supply of vitamin B12 is essential for normal functioning of the brain and the blood. The majority of ALS patients have had insufficient or deficient levels of $25(\mathrm{OH}) \mathrm{D}$ (i.e., calcifediol) at the beginning of the disease. Increasing vitamin D levels can aid mobility and immune control in ALS patients [121]. Folic acid inhibits apoptosis of motoneuronal stem cells, alleviating telomere wear induced by oxidative damage. This effect and other protective effects indicate that supplementation of folic acid may be useful in patients with ALS [122].

\subsection{Ion-channel modulators}

Peripheral nerve hyperexcitability can increase $\mathrm{Ca}^{2+}$ influx into the lower motor neurons, leads to activation of degenerative enzymes, causes mitochondrial dysfunction, produces free radicals, causes deficient production of adenosine triphosphate, and results in the death of motor neurons. As such, hyperexcitability of the peripheral nerves would exacerbate the degenerative process of ALS. Furthermore, overexpression of N-type calcium channels in cortical neurons based on murine models of ALS and the persistent increases in sodium current have been widely demonstrated to determine cortical hyperexcitability in a genetic model of amyotrophic lateral sclerosis. In addition, synaptic interruption and CREB-regulated transcription are restored by $\mathrm{K}^{+}$channel blockers, and it has been observed in other parallel tests that there are anti- $\mathrm{K}^{+}$channel antibodies in certain types of ALS. Hyperexcitability could be a cause or consequence of this autoimmunity in those cases. Further study of these ion channels to discern when and why cortical and peripheral hyperexcitability begins is crucial. Its modulators could correct muscle fiber stiffness in ALS and stabilize motoneuronal action potentials [123, 124, 125, 126].

\subsection{Fluvoxamine/duloxetine}

Cells with high levels of DNA damage associated with senescent muscle fibers stop proliferating and acquire pro-inflammatory properties that contribute to the progression of ALS (i.e., aged muscle that activates p53 in a sustained manner, causing the cells to lose the ability to regenerate). Fluvoxamine/duloxetine inhibits p53 and its apoptotic pathways in peripheral nerves. In addition to its function of modulating p53, it is a high-affinity agonist of the SIGMA1R chaperone that has been shown to be essential in ALS [127]. 
7,8-Dihydroxyflavone is a selective agonist of the receptor tyrosine kinase B (TrkB). As we have discussed in previous sections, it manifests all the therapeutic effects of brain-derived neurotrophic factor (BDNF), such as protecting neurons with damaged mitochondria from apoptosis [64].

\subsection{A diet high in calories, zinc, and hydration}

ALS patients need to compensate for the energy expenditure of hypermetabolism and maintain a high body mass index, which protects against the progression of the disease and from which they can obtain the lipids that will serve as energy for $\beta$-oxidation. They must also maintain adequate hydration, especially during exercise and in warm weather. Additionally, zinc is an essential mineral found especially in the muscles. This mineral is important for cell division, wound healing, blood-sugar regulation, and immune defense. In addition, it participates in the production of testosterone, which is why extra is beneficial for men. Finally, as a cofactor together with copper in the antioxidant enzyme SOD1, we suggest that daily intake of zinc can compensate for environmental copper toxicity.

\subsection{Intense daily muscle physiotherapy plus Clonazepam}

According to our hypothesis, ALS begins at the muscular level; therefore, a continuous exchange of innervation/denervation is necessary so that the negative feedback that from damaged and paralyzed muscle fibers to the MNs does not induce apoptosis. Due to the extremely high plasticity of the nervous system and considering that the brain has been developed for movement, muscles that do not move will end up degenerating the entire neuromotor and vascular system, as the hypertension that patients develop due to lack of muscle movement and the absence of nutrients and oxygen in this tissue will cause a hardening of the walls and changes in the fibroblasts and macrophages in the blood vessels; this has all already been observed in murine models and is characteristic of ALS patients. Physiotherapy would also be beneficial in treating ALS-related muscle cramps, which are sudden, involuntary, and painful contractions. These range from mild cramps that do not affect daily activities or rest, to very severe and intense cramps. Some drugs have been tried to treat cramps in ALS, and we suggest clonazepam (i.e., Rivotril). Likewise, it is necessary to design learning courses on muscle treatment in ALS for physiotherapists, since many may not understand the disease's biochemical landscape.

\section{Concluding remarks and perspectives}

Studies such as those by Barbeito et al. [65] have suggested that molecules such as dichloroacetate (DCA), but also FDA-approved drugs such as ranolazine (RAN) or trimetazidine (TMZ), which inhibit the $\beta$-oxidation of fatty acids, should be reconsidered as therapeutic treatments for ALS due to restoring glycolysis in murine models and exerting positive effects on muscle strength [65]. However, we have questioned whether these positive effects were only momentary and may only occur in certain types of ALS where the necessary glycolytic enzymes and p53 remain in optimal conditions for obtaining glucose. Therefore, we proposed the diversion of glycolysis as a method of obtaining aerobic energy towards $\beta$-oxidation to treat ALS in a broader approach, as this mechanism is, we suspect, compensatory since the beginnings of the disease and could be supported with the administration of daily metformin. This way, NMJs would be intacts. Furthermore, glial inflammation that was generated in the faulty NMJs could be relieved by taking solutex together with metformin such as we have proposed before [9].

ALS is not only a neuronal disease, but it targets MNs, that is, skeletal muscle, which is key during the onset and progression of the disease. In addition, the type of metabolism that muscle fibers develop (i.e., glycolysis versus $\beta$-oxidation) is also critical to its progression. The multiple pathophysiological mechanisms that trigger ALS disease re- 
quire parallel studies. There are many mutated glycolysis and oxidative phosphorylation chain genes in ALS. Sustained, overexcited muscles case glucose consumption due to exhaustion, and their metabolism likely adjusts to obtaining energy from fatty acids. There is an evident change in the metabolism of the glycolytic pathway towards $\beta$-oxidation from the onset of the disease, long before clinical symptoms develop. This may contribute to the increase in oxidative stress since, at a given demand for ATP, the production of cellular fuel from $\beta$-oxidation consumes more oxygen and, subsequently, increase the production of ROS. It would also explain why a high BMI protects against the progression of ALS and would provide a plausible explanation for why ALS patients lose weight rapidly when using the fatty acids mobilized from their adipocytes. More research on muscle energy metabolism is needed to define new therapeutic approaches for the treatment of ALS, particularly those that focus on patients and their medical histories and lifestyle habits as well as primary cell cultures to discern how tissues, both motoneuronal and supportive, are mutually influenced [13].

Magnetic resonance imaging and PET studies in ALS have undoubtedly shown patterns of functional and structural changes considered to be the pathological signatures of the disease. The most advanced neuroimaging techniques should be for all ALS cases to investigate the neurodegenerative cascade and symptoms at a refined and more detailed level. There are still patients who do not receive the benefits of these studies due to the current medical understanding that the physical examination in a consultation and the electromyogram (EMG) are sufficient. Multicenter studies and the implementation of new methodologies could confer a fundamental role to neuroimaging in the clinical setting, accelerating the diagnosis of ALS and allowing rapid prognosis regarding the progression of the disease [128].

ALS has more than 150 years of history. Its incidence remains low, but life expectancy has increased across the board. Still, a healthy person at 30, 40, 50, or even 60 years old can normally expect many more years of life and activity, so when ALS is diagnosed, the scientific and medical community should strive to offer them much more than a paper on which to write their last wishes. ALS is a cruel disease that devastates not just the patient but their entire family. Underlying genetic factors in sporadic ALS, not just in familial ALS, have opened the door to deeper study and exploration of this disease. It is urgent to expand genetic analysis in people with a history of sporadic ALS and in those who are or have been exposed to environments conducive to triggering the disease: Epstein-Barr virus, elite athletes [50], workers in direct contact with toxins or cyanobacteria, people with a family history of diabetes or epilepsy [38, 51, 58, 61, 62], etc. If we can anticipate the diagnosis, we may be able to treat it much earlier, and perhaps one day, to cure it.

Funding: This research received no external funding.

Informed Consent Statement: Not applicable.

Acknowledgments: This review was written after reading more than 200 scientific articles on motor neuron diseases and neurodegeneration, in general, without financial help from public or private funds. We especially want to thank the selfless collaboration of ALS sufferers and their contributions of their confidential medical histories, which have helped us better understand the beginnings of the disease. We also wish to express thanks for the support and clinical information of Jesús Mora Pardina, an expert neurologist in ALS at the San Rafael Hospital in Madrid who, despite being retired, continues to recruit patients from all over the world for clinical trials in Spain, which shows his eagerness to find a cure for the disease. We are grateful for the unconditional help of fellow researchers who have reviewed the article, especially Héctor Preciado, a PhD student, Claremont Graduate University (USA); Jesús Domínguez Manzano from CSIC (Spain); Susana Redondo Gómez, group leader at University of Seville, Spain. 
Conflicts of Interest: The author declares no conflict of interest.

\begin{abstract}
Abbreviations
SOD1: superoxide dismutase 1; TARDBP: DNA binding protein 43 kDa (TDP-43); C9orf72: chromosome 9 open reading frame 72; FUS: fused in sarcoma; ATP: adenosine triphosphate; E2F1: transcription factor E2F1; mTORC1: mammalian target of rapamycin complex 1; BECLIN 1: protein encoded by the BECN1 gene; NMJ: neuromuscular junction; AMPA: alpha-amino-3-hydroxy-5-methyl-4-isoxazolepropionic acid; NMDA: N-methyl-D-aspartate; KO: knockout; SALS: sporadic amyotrophic lateral sclerosis; FALS: familiar amyotrophic lateral sclerosis; qPCR: quantitative PCR; IL-1 $\beta$ : interleukin 1 beta, also known as mononuclear cell factor and lymphocyte activating factor; SPP1: secreted phosphoprotein 1; COL6A1: collagen alpha-1(VI) chain; BMI: body mass index; AMPK: AMP-activated protein kinase; ULK1: Unc-51 like autophagy activating kinase; DNA-PK: DNA-dependent protein kinase; PET: Positron Emission Tomography; CSIC: Consejo Superior de Investigaciones Científicas.
\end{abstract}

\title{
References
}

1. Al-Chalabi, A.; Hardiman, O; Kiernan, M. C.; Chiò, A.; Rix-Brooks, B.; Van den Berg, L. H. Amyotrophic lateral sclerosis: moving towards a new classification system. Lancet Neurol. 2016, 15, 1182-1194. DOI: 10.1016/S1474-4422(16)30199-5.

2. Riancho, J.; Gil-Bea, F. J; Santurtun, A.; López de Munaín, A. Amyotrophic lateral sclerosis: a complex sindrome that needs an integrated research approach. Neural. Regen. Res. 2019, 14, 193-196. DOI: 10.4103/1673-5374.244783

3. Martin, S.; Khleifat, A. A.; Al-Chalabi, A. What causes amyotrophic lateral sclerosis? F1000Res. 2017, 6, 371-381. DOI: 10.12688/f1000research.10476.1.

4. Zhang, J-J.; Zhou, Q-M.; Chen, S.; Le, W-D. Repurposing carbamazepine for the treatment of amyotrophic lateral sclerosis in SOD1-G93A mouse model. CNS Neurosci. Ther. 2018, 24, 1163-1174. DOI: 10.1111/cns.12855.

5. Obrador, E.; Salvador-Palmer, R.; López-Blanch, R.; Jihad-Jebbar, A.; Vallés, S. L.; Estrela, J. M. The Link between Oxidative Stress, Redox Status, Bioenergetics and Mitochondria in the Pathophysiology of ALS. Int. J. Mol. Sci. 2021, 22(12), 6352-6370. DOI: $10.3390 /$ ijms22126352.

6. Mejzini, R.; Flynn, L.L.; Pitout, I.L.; Fletcher, S.; Wilton, S.D.; Akkari, P.A. ALS Genetics, Mechanisms, and Therapeutics: Where Are We Now? Front. in Neurosci. 2019, 13, 1310-1337. DOI: 10.3389/fnins.2019.01310.

7. Volk, A.E.; Weishaupt, J.H.; Andersen, P.M.; Ludolph, A.C.; Kubisch, C. Current knowledge and recent insights into the genetic basis of amyotrophic lateral sclerosis. Med. Genet. 2018, 30, 252-258. DOI: 10.1007/s11825-018-0185-3.

8. Kristensen, R.S.; Bostock, H.; Bronceado, S.V.; Witt, U.; Fuglsang-Frederiksen, U.; Qerama, E.; Andersen, H.; Tankisi, H. MScanFit motor unit number estimation (MScan) and muscle velocity recovery cycle recordings in amyotrophic lateral sclerosis patients. Clin. Neurophysiol. 2019, 130(8), 1280-1288. DOI:10.1016/j.clinph.2019.04.713.

9. Fogarty, M.J.; Amyotrophic lateral sclerosis as a synaptopathy. Neural Regen. Res. 2019, 14(2), 189-192. DOI: 10.4103/1673-5374.244782.

10. Pieri, M.; Carunchio, I.; Curcio, L.; Biagio Mercuri, N.; Zona, C. Increased persistent sodium current determines cortical hyperexcitability in a genetic model of amyotrophic lateral sclerosis. Exp. Neurol. 2009, 215(2), 368-379. DOI: 10.1016/j.expneurol.2008.11.002.

11. Bae, J.S.; Simon, N.G.; Menon, P.; Vucic, S.; Kiernan, M.C. The Puzzling Case of Hyperexcitability in Amyotrophic Lateral Sclerosis. J. Clin. Neurol. 2013, 9 (2), 65-74. DOI:10.3988/jen.2013.9.2.65.

12. Manzano, R.; Toivonen, J.M.; Moreno-Martínez, L.; de la Torre, M.; Moreno-García, L.; López-Royo, T.; Molina, N; Zaragoza, P.; Calvo, A.C.; Osta, R. What skeletal muscle has to say in amyotrophic lateral sclerosis: Implications for therapy. Br. J. Pharmacol. 2020, 178(6), 1279-1297. DOI: 10.1111/bph.15276.

13. Quessada, C.; Bouscary, A; René, F.; Valle, C.; Ferri, A.; Ngo, S.T.; Loefflerj, J.P. Skeletal Muscle Metabolism: Origin or Prognostic Factor for Amyotrophic Lateral Sclerosis (ALS) Development? Cells. 2021, 10(6), 1449. DOI: 10.3390/cells10061449.

14. Singh, T.; Jiao, Y.; Ferrando, L.M.; Yablonska, S.; Li, F.; Horoszko, E.C.; Lacomis, D.; Friedlander, R.M; Carlisle, D.L. Neuronal mitochondrial dysfunction in sporadic amyotrophic lateral sclerosis is developmentally regulated. Scie. Rep. 2021, 11(1), 189316-18932. DOI: 10.1038/s41598-021-97928-7.

15. Premasiri, A.S; Gill, A.L; Vieira, F.G. Type I PRMT Inhibition Protects Against C9ORF72 Arginine-Rich Dipeptide Repeat Toxicity. Front. Pharmacol. 2020, 11, 569661. DOI:10.3389/fphar.2020.569661

16. Bennett, S.A.; Tanaz, R.; Cobos, S.N.; Torrente, M.P. Epigenetics in amyotrophic lateral sclerosis: a role for histone post-translational modifications in neurodegenerative disease. Transl. Res. 2019, 204, 19-30. DOI:10.1016/j.trsl.2018.10.002.

17. Akaishi, T.; Tateyama, M.; Kato, K.; Miura, E.; Izumi, R.; Endo, K.; Sugeno, N.; Suzuki, N.; Baba, T.; Misu, T.; Kikuchi, A.; Hasegawa, T.; Konosu-Fukaya, S.; Fujishima, F.; Suzuki, H.; Nakashima, I.; Aoki, M. An Autopsy Case Involving a 12-year 
History of Amyotrophic Lateral Sclerosis with CIDP-like Polyneuropathy. Inter. Med. 2014, 53(12), $1371-1375$. DOI:10.2169/internalmedicine.53.0774.

18. Echaniz-Laguna MD, A.; Degos MD, B.; Mohr MD, M.; Kessler MD, PhD, R.; Urban-Kraemer MD, E.; Tranchant MD, PhD, C. A study of three patients with amyotrophic lateral sclerosis and a polyneuropathy resembling CIDP. Muscle and Nerve. 2005, 33 (3), 356-362. DOI: 10.1002/mus.20475.

19. Nociti, V.; Frisullo, G.; Marti, A.; Luigetti, M.; Iorio, R.; Patanella, A.K.; Bianco, A.; Tonali, P.A.; Grillo, R.L.; Sabatelli, M.; Batocchi, A.P. Epstein-Barr virus antibodies in serum and cerebrospinal fluid from multiple sclerosis, chronic inflammatory demyelinating polyradiculoneuropathy and amyotrophic lateral sclerosis. J. Neuroimmunol. 2010, 225(1-2), 149-152. DOI:10.1016/j.jneuroim.2010.04.007.

20. Smeyers, J.; Banchi, E.G.; Latouche, M. C9ORF72: What It Is, What It Does, and Why It Matters. Front. Cell. Neurosci. 2021, 15, 661447. DOI:10.3389/fncel.2021.661447.

21. Beckers, J.; Kumar Tharkeshwar, A.; Van Damme, PH. C9orf72 ALS-FTD: recent evidence for dysregulation of the autophagy-lysosome pathway at multiple levels. Autophagy. 2021, 17(11), 3306-3322. DOI:10.1080/15548627.2021.1872189.

22. Castillo, K.; Nassif, M.; Valenzuela, V.; Rojas, F.; Matus, S.; Mercado, G.; Court, F.A.; van Zundert, B.; Hetz, C. Trehalose delays the progression of amyotrophic lateral sclerosis by enhancing autophagy in motoneurons. Autophagy. 2013, 9(9), 1308-1320. DOI: 10.4161/auto.25188.

23. Carreras-Sureda, A.; Jaña, F.; Urra, H.; Durand, S.; Mortenson, D.E.; Sagredo, A.; Bustos, G.; Hazari, Y.; Ramos-Fernández, E.; Sassano, M.L.; Pihán, P.; van Vliet, A.R.; González-Quiroz, M.; Torres, A.K.; Tapia-Rojas, C.; Kerkhofs, M.; Vicente, R.; Kaufman, R.J.; Inestrosa, N.C.; Gonzalez-Billault, C.; Wiseman, R.L.; Agostinis, P.; Bultynck, G.; Court, F.A.; Kroemer, G.; J.Cárdenas, J.C.; Hetz, C. Non-canonical function of IRE1 $\alpha$ determines mitochondria-associated endoplasmic reticulum composition to control calcium transfer and bioenergetics. Nat. Cell Biol. 2019, 21， 755-767. DOI: 10.1038/s41556-019-0329-y.

24. Saleem, A.; Hood, D.A. Acute exercise induces tumour suppressor protein p53 translocation to the mitochondria and promotes a p53-Tfam-mitochondrial DNA complex in skeletal muscle. J. Physiol. 2013, 591(14), 3625-3636. DOI: 10.1113/jphysiol.2013.252791.

25. Jansson, M.; Durant, S.T.; Cho, E-C.; Sheahan, S.; Edelmann, M.; Kessler, B.; La Thangue, N.B. Arginine methylation regulates the p53 response. Nat. Cell Biol. 2008, 10(12), 1431-1439. DOI: 10.1038/ncb1802.

26. Bennett, S.A.; Tanaz, R.; Cobos, S.N.; Torrente, M.P. Epigenetics in amyotrophic lateral sclerosis: a role for histone post-translational modifications in neurodegenerative disease. Transl. Res. 2019, 204, 19-30. DOI:10.1016/j.trsl.2018.10.002.

27. Birbrair, A.; Zhang, T.; Wang, Z-M.; Messi, M.L.; Enikolopov, G.N.; Mintz, A.; Delbono, O. Skeletal muscle neural progenitor cells exhibit properties of NG2-glia. Exp. Cell. Res. 2013, 319(1), 45-63. DOI: 10.1016/j.yexcr.2012.09.008.

28. Itahana, Y.; Itahana, K. Emerging Roles of p53 Family Members in Glucose Metabolism. Int. J. Mol. Sci. 2018, 19(3), 776. DOI: 10.3390/ijms19030776.

29. Beyfuss, K.; Hood, D.A. A systematic review of p53 regulation of oxidative stress in skeletal muscle. Redox Report. 2018, 23(1), 100-117. DOI: 10.1080/13510002.2017.1416773.

30. Yamada, K.; Yoshida, K. Mechanical insights into the regulation of programmed cell death by p53 via mitochondria. Biochim. Biophys. Acta (BBA) - Mol. Cell. Res. 2019, 1866(5), 839-848. DOI: 10.1016/j.bbamcr.2019.02.009.

31. Yeon Kim, Y.; Um, J-H.; Yoon, J-H.; Lee, D-Y.; Jung Lee, Y.; Hyun Kim, D.; Park, J-I.; Yun, J. p53 regulates mitochondrial dynamics by inhibiting Drp1 translocation into mitochondria during cellular senescence. FASEB J. 2020, 34(2), $2451-2464$. DOI: 10.1096/fj.201901747RR.

32. Hoshino, A.; Mita, Y.; Okawa, Y.; Ariyoshi, M.; Iwai-Kanai, E.; Ueyama, T.; Ikeda, K.; Ogata, T.; Matoba, S. Cytosolic p53 inhibits Parkin-mediated mitophagy and promotes mitochondrial dysfunction in the mouse heart. Nat Commun. 2013, 4, 2308. DOI: $10.1038 /$ ncomms3308

33. Martin, L-J. p53 Is Abnormally Elevated and Active in the CNS of Patients with Amyotrophic Lateral Sclerosis. Neurobiol. Dis. 2000, 7(6), 613-622. DOI:10.1006/nbdi.2000.0314.

34. Lahalle, A.; Lacroix, M.; De Blasio, C.; Cissé, M.Y.; Linares, L.K.; Le Cam, L. The p53 Pathway and Metabolism: The Tree That Hides the Forest. Cancers 2021, 13(1), 133. DOI: 10.3390/cancers13010133.

35. Ghavami, S.; Shojaei, S.; Yeganeh, B.; Ande, S.R.; Jangamreddy, J.R.; Mehrpour, M.; Christoffersson, J.; Chaabane, W.; Moghadam, A.R.; Kashani, H.H.; Hashemi, M.; Owji, A.A.; Łos, M.J. Autophagy and apoptosis dysfunction in neurodegenerative disorders. Prog. Neurobiol. 2014, 112, 24-49. DOI: 10.1016/j.pneurobio.2013.10.004.

36. Dunlop, R.A.; Powell, J.T.; Metcalf, J.S.; Guillemin, G.J.; Cox, P.A. L-Serine-Mediated Neuroprotection Includes the Upregulation of the ER Stress Chaperone Protein Disulfide Isomerase (PDI). Neurotox. Res. 2018, 33(1), 113-122. DOI: 10.1007/s12640-017-9817-7

37. Quinn, A.W.; Phillips, C.R.; Violi, J.P.; Steele, J.R.; Johnson, M.S.; Westerhausen, M.T.; Rodgers, K.J. $\beta$-Methylamino-L-alanine-induced protein aggregation in vitro and protection by L-serine. Amino Acids 2021, 53, $1351-1359$. DOI: 10.1007/s00726-021-03049-w.

38. Kung, C-P.; Murphy, M.E. The role of the p53 tumor suppressor in metabolism and diabetes. J. Endocrinol. 2016, 231(2), 61-75. DOI: $10.1530 / \mathrm{JOE}-16-0324$.

39. Maor-Nof, M.; Shipony, Z.; López-González, R.; Nakayama, L.; Zhang, Y-J.; Couthouis, J.; Blum, J.A.; Castruita, P.A.; Linares, G.R.; Ruan, K.; Ramaswami, G.; Simón, D.J.; Nof, A.; Santana, M.; Han, K.; Sinnott-Armstrong, N.; Bassik, M.C.; 
Geschwind, D.H.; Tessier-Lavigne, M.; Attardi, L.D.; Lloyd, T.E.; Ichida, J.K.; Gao, F-B.; Greenleaf, G.J.; Yokoyama, J.S.; Petrucelli, L.; Gitler, A.D. p53 is a central regulator driving neurodegeneration caused by C9orf72 poly(PR). Cell. 2021, 184(3), 689-708. DOI: 10.1016/j.cell.2020.12.025.

40. Lee, J-H.; Jang, H.; Cho, E-J.; Youn, H-D. Ferritin binds and activates p53 under oxidative stress. Biochim. Biophys. Res. Communic. 2009, 389(3), 399-404. DOI: 10.1016/j.bbrc.2009.08.125.

41. Courtney, N.L.; Mole, A.J.; Thomson, A.K.; Murray, L.M. Reduced P53 levels ameliorate neuromuscular junction loss without affecting motor neuron pathology in a mouse model of spinal muscular atrophy. Cell Death $\mathcal{E}$ Disease. 2019, 10(7), 515-529. DOI: 10.1038/s41419-019-1727-6.

42. Martin, L.J.; Wong, M. Skeletal Muscle-Restricted Expression of Human SOD1 in Transgenic Mice Causes a Fatal ALS-Like Syndrome. Front. Neurol. 2020, 11, 592851. DOI: 10.3389/fneur.2020.592851.

43. Ladd, A.C.; Keeney, P.M.; Govind, M.M.; Bennett Jr.J.P. Mitochondrial Oxidative Phosphorylation Transcriptome Alterations in Human Amyotrophic Lateral Sclerosis Spinal Cord and Blood. Neuromol. Med. 2014, 16(4), 714-726. DOI: 10.1007/s12017-014-8321-y.

44. Debska-Vielhaber, G.; Miller, I.; Peeva, V.; Zuschratter, W.; Walczak, J.; Schreiber, S.; Petri, S.; Machts, J.; Vogt, S.; Szczepanowska, J.; Gellerich, F.N.; Hermann, A.; Vielhaber, S.; Kunz, W.S. Impairment of mitochondrial oxidative phosphorylation in skin fibroblasts of SALS and FALS patients is rescued by in vitro treatment with ROS scavengers. Experiment. Neurol. 2021, 339, 113620. DOI: 10.1016/j.expneurol.2021.113620.

45. Lepore, E.; Casola, I.; Dobrowolny, G.; Musarò, A. Neuromuscular Junction as an Entity of Nerve-Muscle Communication. Cells. 2019, 8(8), 906-921. DOI: 10.3390/cells8080906.

46. Dupuis, L.; di Scala, F.; Rene, F.; de Tapia, M.; Oudart, H.; Pradat, P-F.; Meininger, V.; Loeffler, J-P. Up-regulation of mitochondrial uncoupling protein 3 reveals an early muscular metabolic defect in amyotrophic lateral sclerosis. FASEB J. 2003, 17(14), 2091-2093. DOI: 10.1096/fj.02-1182fje.

47. Aráoz 1, R.; Molgó, J.; Tandeau de Marsac, N. Neurotoxic cyanobacterial toxins. Toxicon 2010, 56(5), 813-828. DOI: 10.1016/j.toxicon.2009.07.036.

48. Wang, H.; Xu, C.; Liu, Y.; Jeppesen, E.; Svenning, J-C.; Wu, J.; Zhang, W.; Zhou, T.; Wang, P.; Nangombe, S.; Ma, J.; Duan, H.; de Jingyun, C.; Xie, P. From unusual suspect to serial killer: Cyanotoxins boosted by climate change may jeopardize megafauna. The Innovation. 2021, 2(2), 100092. DOI:10.1016/j.xinn.2021.100092.

49. Masseret, E.; Banack, S.; Boumédiène, F.; Abadie, E.; Brient, L.; Pernet, F.; Juntas-Morales, R.; Pageot, N.; Metcalf, J.; Cox, P.; Camu, W. Dietary BMAA Exposure in an Amyotrophic Lateral Sclerosis Cluster from Southern France. Plos One 2013, 8(12), 83406. DOI: 10.1371/journal.pone.0083406.

50. Daneshva, D.H.; Mez, J.; Alosco, M.L.; Baucom, Z.H.; Mahar, I.; Baug, C.M.; Valle, J.P.; Weuve, J.; Paganoni, S.; Cantu, R.C.; Zafonte, R.D.; Stern, R.A.; Stein, T.D.; Tripodis, Y.; Nowinski, C.J.; McKee, A.C. Incidence of and Mortality From Amyotrophic Lateral Sclerosis in National Football League Athletes. JAMA Netw. Open. 2021, 4(12), 2138801. DOI: 10.1001/jamanetworkopen.2021.38801.

51. Tefera, T. W.; Tan, K. N.; McDonald, T. S.; Borges, K. Alternative Fuels in Epilepsy and Amyotrophic Lateral Sclerosis. Neurochem Res. 2017, 42(6), 1610-1620. DOI: 10.1007/s11064-016-2106-7.

52. Principal, B.J.; Dunlop, R.A.; Rodgers, K.J. The use of 1-serine to prevent $\beta$-methylamino-l-alanine (BMAA)-induced proteotoxic stress in vitro. Toxicon 2016, 109, 7-12. DOI: 10.1016/j.toxicon.2015.11.003.

53. Sini, P.; Chau Dang, T.B.; Fais, M.; Galioto, M.; Padedda, B.M.; Lugliè, A.; Iaccarino, C.; Crosio, C. Cyanobacteria, Cyanotoxins, and Neurodegenerative Diseases: Dangerous Liaisons. Int. J. Mol. Sci. 2021, 22(16), 8726. DOI: 10.3390/ijms22168726.

54. Zhang, Y.; Gu, J.; Sun, Q. Aberrant Stress Granule Dynamics and Aggrephagy in ALS Pathogenesis. Cells. 2021, 10, 2247. DOI: 10.3390/cells10092247.

55. Kumar Jha, M.; Jeon, S.; Suk, K. Pyruvate Dehydrogenase Kinases in the Nervous System: Their Principal Functions in Neuronal-glial Metabolic Interaction and Neuro-metabolic Disorders. Curr. Neuropharmacol. 2012, 10(4), 393-403. DOI: 10.2174/157015912804143586.

56. Aleshin, V.A.; Artiukhov, A.V.; Kaehne, T.; Graf, A.V.; Bunik, V.I. Daytime Dependence of the Activity of the Rat Brain Pyruvate Dehydrogenase Corresponds to the Mitochondrial Sirtuin 3 Level and Acetylation of Brain Proteins, All Regulated by Thiamine Administration Decreasing Phosphorylation of PDHA Ser293. Int. J. Mol. Sci. 2021, 22(15), 8006-8024. DOI: 10.3390/ijms22158006.

57. Anwar, S.; Shamsi, A.; Mohammad, T.; Islam, A.; Hassan, Md.I. Targeting pyruvate dehydrogenase kinase signaling in the development of effective cancer therapy. Biochim. Biophys. Acta (BBA) Rev. Cancer. 2021, 1876(1), 188568. DOI: 10.1016/j.bbcan.2021.188568.

58. Prasad, C.; Rupar, T.; Prasad, A.N. Pyruvate dehydrogenase deficiency and epilepsy. Brain Devel. 2011, $33(10), 856-865$. DOI: 10.1016/j.braindev.2011.08.003.

59. Goncharova, P.S.; Davydova, T.K.; Popova, T.E.; Novitsky, M.A.; Petrova, M.M.; Gavrilyuk, O.A.; Al-Zamil, M.; Zhukova, N.G.; Nasyrova, R.F.; Shnayder, N.A. Nutrient Effects on Motor Neurons and the Risk of Amyotrophic Lateral Sclerosis. Nutrients. 2021, 13(11), 3804. DOI: 10.3390/nu13113804. 
60. Paoli, A.; Bianco, A.; Damiani, E.; Bosco, G. Ketogenic Diet in Neuromuscular and Neurodegenerative Diseases. BioMed Res. Internat. 2014, 474296. DOI: 10.1155/2014/474296.

61. Vasta, R.; D'Ovidio, F., Logroscino, G.; Chiò, A. The links between diabetes mellitus and amyotrophic lateral sclerosis. Neurol. Sci. 2021, 42(4), 1377-1387. DOI: 10.1007/s10072-021-05099-0.

62. 62. Ferri, L.; Ajdina1, P.; Gabriella Rispoli, M.; Carrarini, C.; Barbone, F.; D'Ardes, D.; Capasso, M.; Di Muzio, A.; Cipollone, F.; Onofrj, M.; Bonanni, L. Diabetes Mellitus and Amyotrophic Lateral Sclerosis: A Systematic Review. Biomolecules. 2021, 11(6), 867. DOI: 10.3390/biom11060867.

63. Sundaramoorthy, V.; Walker, A.K.; Tan, V.; Fifita, J.A.; Mccann, E.P.; Williams, K.L.; Blair, I.P.; Guillemin, G.J.; Farg, M.A.; Atkin, J.D. Defects in optineurin- and myosin VI-mediated cellular trafficking in amyotrophic lateral sclerosis. Hum. Mol. Gen. 2015, 24(13), 3830-3846. DOI: 10.1093/hmg/ddv126.

64. Korkmaz, O.T.; Aytan, N.; Carreras, I.; Choi, J-K.; Kowall, N.W.; Jenkins, B.G.; Dedeoglu, A. 7,8-Dihydroxyflavone improves motor performance and enhances lower motor neuronal survival in a mouse model of amyotrophic lateral sclerosis. Neurosci. Lett. 2014， 566， 286-291. DOI: 10.1016/j.neulet.2014.02.058.

65. Martínez-Palma, L.; Miquel, E.; Lagos-Rodríguez, V.; Barbeito, L.; Cassina, A.; Cassina, P. Correction to: Mitochondrial Modulation by Dichloroacetate Reduces Toxicity of Aberrant Glial Cells and Gliosis in the SOD1G93A Rat Model of Amyotrophic Lateral Sclerosis. Neurotherap. 2020, 17(4), 2089. DOI: 10.1007/s13311-020-00872-z.

66. Smith, E.F.; Shaw, P.J.; De Vos, K.J. The role of mitochondria in amyotrophic lateral sclerosis. Neurosci. Lett. 2019, 710, 132933. DOI: 10.1016/j.neulet.2017.06.052.

67. David, S.; López-Vales, R. Bioactive Lipid Mediators in the Initiation and Resolution of Inflammation after Spinal Cord Injury. Neurosci. 2021, 466, 273-297. DOI: 10.1016/j.neuroscience.2021.04.026.

68. Rosbashunb, M. Metformin treatment of the C9orf72 ALS/FTD mouse: Almost too good for words. Proc. Natl. Acad. Sci. USA. 2020, 117(33), 19627-19628. DOI: 10.1073/pnas.2012363117.

69. Leal-Lasarte, M.; Mannini, B.; Chiti, F.; Vendruscolo, M.; Dobson, C.M.; Roodveldt, C.; Pozo, D. Distinct responses of human peripheral blood cells to different misfolded protein oligomers. Immunol. 2021, 164, 358-371. DOI: 10.1111/imm.13377.

70. Brillante, F.; Chan, G.; van Hummel, A.; Ittner, L.M.; Ke, Y.D. TDP-43 and Inflammation: Implications for Amyotrophic Lateral Sclerosis and Frontotemporal Dementia. Int. J. Mol. Sci. 2021, 22, 7781-7806. DOI: 10.3390/ijms22157781.

71. Nwosu, V.K.; Royer, J.A.; Stickler, D.E. Voltage gated potassium channel antibodies in amyotrophic lateral sclerosis. Amyotrophic Lateral Sclerosis 2010, 11, 392-394. DOI: 10.3109/17482960903452283.

72. Zhao, Y.; Qiu, W.; Liu 1, J.; Yuan, X.; Mao, W.; Yin, J.; Peng, B.; Liu, W.; Han, S.; He, X. Blockade of Kv1.3 potassium channel inhibits CD8 $+\mathrm{T}$ cell-mediated neuroinflammation via PD-1/Blimp-1 signaling. FASEB J. 2020, 34(11), 15492-15503. DOI: 10.1096/fj.202000861RR.

73. Kim, S.H.; Koh, J.H.; Higashida, K.; Jung, S.R.; Holloszy, J.O.; Han, D-H. PGC-1 $\alpha$ mediates a rapid, exercise-induced downregulationof glycogenolysis in rat skeletal muscle. J. Physiol. 2015, 593(3), 635. DOI: 10.1113/jphysiol.2014.283820.

74. Patron, M.; Checchetto, V.; Raffaello, A.; Teardo, E.; Vecellio Reane, D.; Mantoan, M.; Granatiero, V.; Szabò, I.; De Stefani, D.; Rizzuto, R. MICU1 and MICU2 Finely Tune the Mitochondrial Ca2+ Uniporter by Exerting Opposite Effects on MCU Activity. Mol Cell. 2014, 53 (5), 726-737. DOI: 10.1016/j.molcel.2014.01.013.

75. Ibarra García Padilla, R. Behavior of the mitochondrial calcium uniporter modulated by antagonistic elements. Rev. Educ. Bioquím. 2014, 33(4), 1-19. DOI: 10.1371/journal.pone.0142005.

76. Dafinca, R.; Barbagallo, P.; Farrimond, L.; Candalija, A.; Scaber, J.; Ababneh, N.A.; Sathyaprakash, C.; Vowles, J.; Cowley, S.A.; Talbot, K. Impairment of Mitochondrial Calcium Buffering Links Mutations in C9ORF72 and TARDBP in iPS-Derived Motor Neurons from Patients with ALS/FTD. Stem Cell Report 2020, 14(5), 892-908. DOI: 10.1016/j.stemcr.2020.03.023.

77. Matteucci, A.; Patron, M.; Vecellio Reane, D.; Gastaldello, S.; Amoroso, S.; Rizzuto, R.; Brini, M.; Raffaello, A.; Cali, T. Parkin-dependent regulation of the MCU complex component MICU1. Scientific Report. 2018, 8(1), 32551-32557. DOI: 10.1038/s41598-018-32551-7.

78. Watanabe, S.; Ilieva, H.; Tamada, H.; Nomura, H.; Komine, O.; Endo, F.; Jin, S.; Mancias, P.; Kiyama, H.; Yamanaka, K. Mitochondria-associated membrane collapse is a common pathomechanism in SIGMAR1- and SOD1-linked ALS. EMBO Mol. Med. 2016, 8(12), 1421-1437. DOI: 10.15252/emmm.201606403

79. Borgese, N.; Iacomino, N.; Colombo, S.F.; Navone, F. The Link between VAPB Loss of Function and Amyotrophic Lateral Sclerosis. Cells. 2021, 10(8), 1865. DOI: 10.3390/cells10081865.

80. Jesse, C.M.; Bushuven, E.; Tripathi, P.; Chandrasekar, A.; Simón, C.M.; Drepper, C.; Yamoah, A.; Dreser, A.; Katona, I.; Johann, S.; Beyer, C.; Wagner, S.; Grond, M.; Nikolin, S.; Anink, J.; Troost, D.; Sendtner, M.; Goswami, A.; Weis, J. ALS-Associated Endoplasmic Reticulum Proteins inDenervated Skeletal Muscle: Implications for Motor Neuron Disease Pathology. Brain Pathol. 2017, 27(6), 781-794. DOI:10.1111/bpa.12453.

81. Sakai, S.; Watanabe, S.; Komine, O.; Sobue, A.; Yamanaka, K. Novel reporters of mitochondria-associated membranes (MAM), MAMtrackers, demonstrate MAM disruption as a common pathological feature in amyotrophic lateral sclerosis. FASEB J. 2021, 35(7), 21688-21702. DOI: 10.1096/fj.202100137R.

82. Rieusset, J. Role of Endoplasmic Reticulum-Mitochondria Communication in Type 2 Diabetes. Adv. Exp. Med. Biol. 2017, 997, 171-186. DOI: 10.1007/978-981-10-4567-7_13. 
83. Lautenschläger, J.; Prell, T.; Ruhmer, J.; Weidemann, L.; Witte, O.W.; Grosskreutz, J. Overexpression of human mutated G93A SOD1 changes dynamics of the ER mitochondria calcium cycle specifically in mouse embryonic motor neurons. Experiment. Neurol. 2013, 247, 91-100. DOI: 10.1016/j.expneurol.2013.03.027.

84. Zhemkov, V.; Geva, M.L.; Hayden, M.R.; Bezprozvanny, I. Sigma-1 Receptor (S1R) Interaction with Cholesterol: Mechanisms of S1R Activation and Its Role in Neurodegenerative Diseases. Int. J. Mol. Sci. 2021, 22(8), 4082. DOI: 10.3390/ijms22084082.

85. Zhemkov, V.; Ditlev, J.A.; Lee, W-R.; Wilson, M.; Liou, J.; Rosen, M.K.; Bezprozvanny, I. The role of sigma 1 receptor in organization of endoplasmic reticulum signaling microdomains. ELife. 2021, 10, 65192. (2021). DOI: 10.7554/eLife.65192.

86. Mavlyutov, T.A.; Baker, E.M.; Losenegger, T.M.; Kim, J.R.; Torres, B.; Epstein, M.L.; Ruoho, A.E. The Sigma-1 Receptor-A Therapeutic Target for the Treatment of ALS? Adv. Exp. Med. Biol. 2017, 964, 255-265. DOI: 10.1007/978-3-319-50174-1_17.

87. Zhang, X.; Chen, S.; Lu, K.; Wang, F.; Deng, J.; Xu, Z.; Wang, X.; Zhou, Q.; Le, W.; Zhao, Y. Verapamil Ameliorates Motor Neuron Degeneration and Improves Lifespan in the SOD1G93A Mouse Model of ALS by Enhancing Autophagic Flux. Aging Disease. 2019, 10(6), 1159-1173. DOI: 10.14336/AD.2019.0228.

88. Tagashira, H.; Shinoda, Y.; Shioda, N.; Fukunaga, K. Methyl pyruvate rescues mitochondrial damage caused by SIGMAR1 mutation related to amyotrophic lateral sclerosis. Biochim. Biophys. Acta (BBA). 2014, 12, 3320-3334. DOI: 10.1016/j.bbagen.2014.08.012.

89. Crouzier, L.; Denus, M.; Richard, E.M.; Tavernier, A.; Diez, C.; Cubedo, N.; Mauricio, T.; Delprat, B. Sigma-1 Receptor Is Critical for Mitochondrial Activity and Unfolded Protein Response in Larval Zebrafish. Int. J. Mol. Sci. 2021, 22(20), 11049-11068. DOI: 10.3390/ijms222011049.

90. Yao, R-Q.; Ren, C.; Xia, Z-F.; Yao, Y-M. Organelle-specific autophagy in inflammatory diseases: a potential therapeutic target underlying the quality control of multiple organelles. Autophagy. 2021, 17(2), 385-401. DOI: 10.1080/15548627.2020.1725377.

91. Xu, Y.; Shen, J.; Ran, Z. Emerging views of mitophagy in immunity and autoimmune diseases. Autophagy. 2020, 16(1), 3-17. DOI: 10.1080/15548627.2019.1603547.

92. Deora, V.; Lee, J.D.; Albornoz, E.A.; McAlary, L.; Jagaraj, C.J.; Robertson, A.A.B.; Atkin, J.D.; Cooper, M.A.; Schroder, K.; Yerbury, J.J.; Gordon, R.; Woodruff, T.M. The microglial NLRP3 inflammasome is activated by amyotrophic lateral sclerosis proteins. Glia. 2020, 68(2), 407-421. DOI: 10.1002/glia.23728.

93. Rai, P.; Janardhan, K. S.; Meacham, J.; Madenspacher, J. H.; Lin, W-C; Karmaus, P. W.F.; Martinez, J.; Li, Q-Z.; Yan, M.; Zeng, J.: Grinstaff, M. W.; Shirihai, O. S.; Taylor, G. A.; Fessler, M. B. IRGM1 links mitochondrial quality control to autoimmunity. Nat Immunol. 2021, 22(3), 312-321. DOI: 10.1038/s41590-020-00859-0.

94. Sevenich, L. Brain-Resident Microglia and Blood-Borne Macrophages Orchestrate Central Nervous System Inflammation in Neurodegenerative Disorders and Brain Cancer. Front. Immunol. 2018, 9, 697. DOI: 10.3389/fimmu.2018.00697.

95. Gugliandolo, A.; Giacoppo, S.; Bramanti, P.; Mazzon, E. NLRP3 Inflammasome Activation in a Transgenic Amyotrophic Lateral Sclerosis Model. Inflammat. 2018, 41, 93-103. DOI: 10.1007/s10753-017-0667-5.

96. Stallings, N.R.; Puttaparthi, K.; Dowling, K.J.; Lutero, C.M.; Burns, D.K.; Davis, K.; Elliott, J.L. TDP-43, an ALS Linked Protein, Regulates Fat Deposition and Glucose Homeostasis. Plos One. 2013, 8(8), 71793. DOI: 10.1371/journal.pone.0071793.

97. Vogt, M.A.; Ehsaei, Z.; Knuckles, P.; Higginbottom, A.; Helmbrecht, M.S.; Kunath, T.; Eggan, K.; Williams, L.A.; Shaw, P.J.; Wurst, W.; Floss, T.; Huber, A.B.; Taylor, V. TDP-43 induces p53-mediated cell death of cortical progenitors and immature neurons. Sci. Rep. 2018, 8, 8097. DOI: 10.1038/s41598-018-26397-2.

98. Jiang, P.; Du, W.; Wang, X.; Mancuso, A.; Gao, X.; Wu, M.; Yang, X. p53 regulates biosynthesis through direct inactivation of glucose-6-phosphate dehydrogenase. Nat. Cell Biol. 2011, 13(3), 310-316. DOI: 10.1038/ncb2172.

99. Gomes, A.S.; Ramos, H.; Soares, J.; Saraiva, L. p53 and glucose metabolism: an orchestra to be directed in cancer therapy. Pharmacol. Res. 2018, 131, 75-86. DOI: 10.1016/j.phrs.2018.03.015.

100. Ding, X-1.; Lei, P. Plasma Replacement Therapy for Alzheimer's Disease. Neurosci. Bu. 2020, 36, 89-90. DOI: 10.1007/s12264-019-00394-5.

101. Boada, M.; Martínez-Lage, P.; Serrano-Castro, P.; Costa, M.; Páez, A. Therapeutic plasma exchange with albumin: a new approach to treat Alzheimer's disease. Expert Rev. Neurotherap. 2021, 21(8), 843-849. DOI: 10.1080/14737175.2021.1960823.

102. Silani, V.; Scarlato, G.; Valli, G.; Marconi, M. Plasma Exchange Ineffective in Amyotrophic Lateral Sclerosis. Arch. Neurol. 1980, 37(8), 511-513. DOI: 10.1001/archneur.1980.00500570059009.

103. Milligan, C.; Atassi, N.; Babu, S.; Barohn, R.J.; Caress, J.B.; Cudkowicz, M.E.; Evora, A.; Hawkins, G.A.; Wosiski-Kuhn, M.; Macklin, E.A.; Shefner, J.M.; Simmons, Z.; Bowser, R.P.; Ladha, S.S. Tocilizumab is safe and tolerable and reduces C-reactive protein concentrations in the plasma and cerebrospinal fluid of ALS patients. Muscle Nerve. 2021, 64(3), 309-320. DOI: 10.1002/mus.27339.

104. Månberg, A.; Skene, N.; Sanders, F.; Trusohamn, F.; Remnestål, J., Szczepińska, A.; Aksoylu, L.S.; Lönnerberg, P.; Ebarasi, L.; Wouters, S.; Lehmann, M.; Olofsson, J.; von Gohren Antequera, I.; Domaniku, A.; De Schaepdryver, M.; De Vocht, J.; Poesen, K.; Uhlén, M.; Anink, J.; Mijnsbergen, C.; Vergunst-Bosch, H.; Hübers, A.; Kläppe, U.; Rodri- 
guez-Vieitez, E.; Gilthorpe, J.D.; Hedlund, E.; Harris, R.A.; Aronica, E.; Van Damme, P.; Ludolph, A.; Veldink, J., Ingre, C.; Nilsson, P.; Lewandowski, S.A. Altered perivascular fibroblast activity precedes ALS disease onset. Nat. Med. 2021, 27, 640-646. DOI: 10.1038/s41591-021-01295-9.

105. Dorrier, C.E.; Jones, H.E.; Pintarić, L.; Siegenthaler, J.A.; Daneman, R. Emerging roles for CNS fibroblasts in health, injury and disease. Nat. Rev. Neurosci. 2022, 23(1), 23-34. DOI: 10.1038/s41583-021-00525-w.

106. Uaesoontrachoon, K.; Yoo, H-J.; Tudor, E.M.; Pike, R.N.; Mackie, E.J.; Pagel, C.N. Osteopontin and skeletal muscle myoblasts: Association with muscle regeneration and regulation of myoblast function in vitro. Int. J. Biochem. Cell Biol. 2008, 40(10), 2303-2314. DOI: 10.1016/j.biocel.2008.03.020.

107. Vetrone, S.A.; Montecino-Rodriguez, E.; Kudryashova, E.; Kramerova, I.; Hoffman, E.P.; Liu, S.D.; Miceli, M.C.; Spencer, M.J. Osteopontin promotes fibrosis in dystrophic mouse muscle by modulating immune cell subsets and intramuscular TGF- $\beta$. J. Clin. Invest. 2009, 119(6), 1583-1594. DOI: 10.1172/JCI37662.

108. Patel, P.; Julien, J-P.; Kriz, J. Early-Stage Treatment with Withaferin A Reduces Levels of Misfolded Superoxide Dismutase 1 and Extends Lifespan in a Mouse Model of Amyotrophic Lateral Sclerosis. Neurotherap. 2015, 12(1), 217-233. DOI: 10.1007/s13311-014-0311-0.

109. Landmark, C.J. Antiepileptic Drugs in Non-Epilepsy Disorders. Relations between Mechanisms of Action and Clinical Efficacy. CNS Drugs. 2008, 22, 27-47. DOI: 10.2165/00023210-200822010-00003.

110. Zhang, J-J.; Zhou, Q-M.; Chen, S.; Le, W-D. Repurposing carbamazepine for the treatment of amyotrophic lateral sclerosis in SOD1-G93A mouse model. CNS Neurosci. Ther. 2018, 24, 1163-1174. DOI: 10.1111/cns.12855.

111. Debska-Vielhaber, G.; Miller, I.; Peeva, V.; Zuschratter, W.; Walczak, Schreiber, J.S.; Petri, S.; Machts, J.; Vogt, S.; Szczepanowska, J.; Gellerich, F.N.; Hermann, A.; Vielhaber, S.; Kunz, W.S. Impairment of mitochondrial oxidative phosphorylation in skin fibroblasts of SALS and FALS patients is rescued by in vitro treatment with ROS scavengers. Experiment. Neurol. 2021, 339, 113620. DOI: 10.1016/j.expneurol.2021.113620.

112. Yamano, K.; Kikuchi, R.; Kojima, W.; Hayashida, R.; Koyano, F.; Kawawaki, J.;Shoda, T.; Demizu, Y.; Naito, M.; Tanaka, K.; Matsuda, N. Critical role of mitochondrial ubiquitination and the OPTN-ATG9A axis in mitophagy. J. Cell Biol. 2020, 219(9), 201912144. DOI: $10.1083 /$ jcb.201912144.

113. Richter, B.D. Sliter, A.; Herhaus, L.; Stolz, A.; Wang, C.; Beli, P.; Zaffagnini, G.; Wild, P.; Martens, S.; Wagner, S.A.; Youle, R.J.; Dikic, I. Phosphorylation of OPTN by TBK1 enhances its binding to Ub chains and promotes selective autophagy of damaged mitochondria. Proc. Natl. Acad. Sci. USA. 2016, 113(15), 4039-4044. DOI: 10.1073/pnas.1523926113.

114. O'Loughlin, T.; Kruppa, A.J.; Ribeiro, A.L.R.; Edgar, J.R.; Ghannam, A.; M. Smith, A.; Buss, F. OPTN recruitment to a Golgi-proximal compartment regulates immune signalling and cytokine secretion. J. Cell Sci. 2020, 133(12), 239822. DOI:10.1242/jcs.239822.

115. Akizuki, M.; Yamashita, H.; Uemura, K.; Maruyama, H.; Kawakami, H.; Ito, H.; Takahashi,R. Optineurin suppression causes neuronal cell death via NF-кB pathway. J. Neurochem. 2013, 126(6), 699-704. DOI: 10.1111/jnc.12326.

116. Madruga, E.; Maestro, I.; Martínez, A. Mitophagy Modulation, a New Player in the Race against ALS. Int. J. Mol. Sci. 2021, 22(2), 740-761. DOI: 10.3390/ijms22020740.

117. Jin Tak, Y.; Park, J-H.; Rhim, H.; Kang, S. ALS-Related Mutant SOD1 Aggregates Interfere with Mitophagy by Sequestering the Autophagy Receptor Optineurin. Int. J. Mol. Sci. 2020, 21(20), 7525-7542. DOI: 10.3390/ijms21207525.

118. Castillo, K.; Nassif, M.; Valenzuela, V.; Rojas, F.; Matus, S.; Mercado, G.; Court, F.A.; van Zundert, B.; Hetz, C. Trehalose delays the progression of amyotrophic lateral sclerosis by enhancing autophagy in motoneurons. Autophagy. 2013, 9(9), 1308-1320. DOI: 10.4161/auto.25188.

119. Castiglioni, C.; Verrigni, D.; Okuma, C.; DIaz, A.; Alvarez, K.; Rizza, T.; Carrozzo, R.; Bertini, E.; Miranda, M. Pyruvate dehydrogenase deficiency presenting as isolated paroxysmal exercise induced dystonia successfully reversed with thiamine supplementation. Case report and mini-review. Eur. J. Paedriat. Neurol. 2015, 19(5), 497-503. DOI: 10.1016/j.ejpn.2015.04.008.

120. Bald, E.M.; Nance, C.S.; Schultz, J.L. Melatonin may slow disease progression in amyotrophic lateral sclerosis: Findings from the Pooled Resource Open-Access ALS Clinic Trials database. Muscle Nerve. 2021, 63(4), 572-576. DOI: 10.1002/mus.27168.

121. Paganoni, S.; Macklin, E.A.; Karam, C.; Yu, H.; Gonterman, F.; Ashley Fetterman, K.; Cudkowicz, M.; Berry, J.; Wills, A-M. Vitamin D levels are associated with gross motor function in amyotrophic lateral sclerosis (Clinical Research). Muscle and Nerve. 2017, 56(4), 726-731. DOI: 10.1002/mus.25555.

122. Li, Z.; Li, W.; Zhou, D.; Zhao, J.; Ma, Y.; Huang, L.; Dong, C.; Wilson, J.X.; Huang, G. Alleviating Oxidative Damage-Induced Telomere Attrition: a Potential Mechanism for Inhibition by Folic Acid of Apoptosis in Neural Stem Cells. Mol Neurobiol. 2022, 59, 590-602. DOI: 10.1007/s12035-021-02623-3.

123. Iwai, Y.; Shibuya, K.; Misawa, S.; Sekiguchi, Y.; Watanabe, K.; Amino, H.; Kuwabara, S. Axonal Dysfunction Precedes Motor Neuronal Death in Amyotrophic Lateral Sclerosis. Plos One. 2016, 11(7), 1-9. DOI: 10.1371/journal.pone.0158596.

124. Catanese, A.; Rajkumar, S.; Sommer, D.; Freisem, D.; Wirth, A.; Aly, A.; Massa-López, D.; Olivieri, A.; Torelli, F.; Ioannidis, V.; Lipecka, J.; Chiara Guerrera, I.; Zytnicki, D.; Ludolph, A.; Kabashi, E.; Mulaw, M.A.; Roselli, F.; Böckers, T.M Synaptic disruption and CREB-regulatedtranscription are restored by K+channel blockersin ALS. EMBO Mol. Med. 2021, 13(7), 13131 DOI: $10.15252 / \mathrm{emmm} .202013131$. 
125. Pieri, M.; Carunchio, I.; Curcio, L.; Biagio Mercuri, N.; Zona, C. Increased persistent sodium current determines cortical hyperexcitability in a genetic model of amyotrophic lateral sclerosis. Exp. Neurol. 2009, 215(2), 368-379. DOI: 10.1016/j.expneurol.2008.11.002.

126. Pieri, M.; Caioli, S.; Canu, N.; Mercuri, N.B.; Guatteo, E.; Zona, C. Over-expression of N-type calcium channels in cortical neurons from a mouse model of Amyotrophic Lateral Sclerosis. Exp. Neurol. 2013, 247, 349-358. DOI: 10.1016/j.expneurol.2012.11.002.

127. Maor-Nof, M.; Shipony, Z.; López-González, R.; Nakayama, L.; Zhang, Y-J.; Couthouis, J.; Blum, J.A.; Castruita, P.A.; Linares, G.R.; Ruan, K.; Ramaswami, G.; Simón, D.J.; Nof, A.; Santana, M.; Han, K.; Sinnott-Armstrong, N.; Bassik, M.C.; Geschwind, D.H.; Tessier-Lavigne, M.; Attardi, L.D.; Lloyd, T.E.; Ichida, J.K.; Gao, F-B.; Greenleaf, G.J.; Yokoyama, J.S.; Petrucelli, L.; Gitler, A.D. p53 is a central regulator driving neurodegeneration caused by C9orf72 poly (PR). Cell. 2021, 184(3), 689-708. DOI: 10.1016/j.cell.2020.12.025.

128. Kassubek, J.; Pagani, M. Imaging in amyotrophic lateral sclerosis: MRI and PET. Curr. Opin. Neurol. 2019, 32(5), 740-746. DOI: 10.1097/WCO.0000000000000728. 